\title{
SEPARATION PROPERTIES IN THE PRIMITIVE IDEAL SPACE OF A MULTIPLIER ALGEBRA
}

\author{
ROBERT J. ARCHBOLD AND DOUGLAS W.B. SOMERSET
}

\begin{abstract}
We use recent work on spectral synthesis in multiplier algebras to give an intrinsic characterization of the separable $\mathrm{C}^{*}$-algebras $A$ for which $\operatorname{Orc}(M(A))=1$, i.e. for which the relation of inseparability on the topological space of primitive ideals of the multiplier algebra $M(A)$ is an equivalence relation. This characterization has applications to the calculation of norms of inner derivations and other elementary operators on $A$ and $M(A)$. For example, we give necessary and sufficient conditions on the ideal structure of a separable $\mathrm{C}^{*}$-algebra $A$ for the norm of every inner derivation to be twice the distance of the implementing element to the centre of $M(A)$.
\end{abstract}

2000 Mathematics Subject Classification: 46L05, 46L07, 46L57 (primary), 54D15, 54D35, 54G05 (secondary).

\section{INTRODUCTION}

The origin of the paper lies in the following problem: suppose that $A$ is a non-unital $C^{*}$-algebra and that $\operatorname{Prim}(A)$, the primitive ideal space of $A$ with the hull-kernel topology, has some topological property. In what circumstances does $\operatorname{Prim}(M(A))$ (where $M(A)$ is the multiplier algebra of $A$ ) inherit the same or a similar property? For instance, if $\operatorname{Prim}(A)$ is Hausdorff, when is $\operatorname{Prim}(M(A))$ Hausdorff? This question is non-trivial, even in the simplest case when $A$ is $n$-homogeneous, and in Theorem 2.1 we give an example of a 2-homogeneous $\mathrm{C}^{*}$-algebra $A$ for which $\operatorname{Prim}(M(A))$ is non-Hausdorff.

Although Prim $(M(A))$ in Theorem 2.1 is non-Hausdorff, it does have the property that the relation $\sim$ on $\operatorname{Prim}(M(A))$ is an equivalence relation (where for a $\mathrm{C}^{*}$-algebra $B$ we say that $P \sim Q$ if the primitive ideals $P$ and $Q$ cannot be separated by disjoint open sets in $\operatorname{Prim}(B))$. In general the relation $\sim$ is reflexive and symmetric but not necessarily transitive. When $\sim$ is an equivalence relation on $\operatorname{Prim}(A)$ we say that $\operatorname{Orc}(A)=1$, and otherwise the connecting order $\operatorname{Orc}(A)$ is greater than 1 (for an explanation of this terminology, see [40]). $\mathrm{C}^{*}$-algebras with $\operatorname{Orc}(A)=1$ include von Neumann and $\mathrm{AW}^{*}$-algebras and their quotients, numerous group $\mathrm{C}^{*}$-algebras (see Section 3 ), the spoke-algebras of [13], and also quasi-standard $\mathrm{C}^{*}$ algebras (i.e. those for which the relation $\sim$ is an open equivalence relation on $\operatorname{Prim}(A)$ $[9])$.

The main purpose of this paper is to characterize the separable $\mathrm{C}^{*}$-algebras $A$ for which $\operatorname{Orc}(M(A))=1$. Since $\operatorname{Prim}(A)$ is canonically homeomorphic to an open subset of $\operatorname{Prim}(M(A))[37,4.1 .10]$, it is easily seen that such algebras necessarily have $\operatorname{Orc}(A)=1$. Regarding $\mathrm{A}$ as a $C_{0}(X)$-algebra in a natural way, we show in Theorem 4.2 that a necessary condition for $\operatorname{Orc}(M(A))=1$ is that $M(A)$ should have spectral synthesis as a $C(\beta X)$-algebra in the sense of [12] (where $\beta X$ is the Stone-Čech compactification of $X$ ). 
Combining this with the characterization of spectral synthesis in [12, Corollary 3.10], it follows that $\operatorname{Orc}(M(A))=1$ if and only if $\operatorname{Orc}(A)=1$ and the Glimm ideals of $A$ are locally modular (Corollary 4.7).

Part of our interest in the condition $\operatorname{Orc}(A)=1$ arises from its connection with the derivation constant $K_{s}(A)$ (see Section 5). Indeed, if $A$ is a unital non-commutative $C^{*}$ algebra, $K_{s}(A)$ takes the optimal value $1 / 2$ if and only if $\operatorname{Orc}(A)=1$ [40, Theorem 4.4]. Thus Corollary 4.7 allows us to characterize the separable $\mathrm{C}^{*}$-algebras $A$ for which $K_{s}(M(A))=$ 1/2 (Corollary 5.1).

We now describe the structure of the paper. In Section 2 we give the example of a 2 homogeneous $C^{*}$-algebra $A$ such that $\operatorname{Prim}(M(A))$ is non-Hausdorff. In Section 3, we solve an old problem for $\sigma$-unital $C^{*}$-algebras with $\operatorname{Orc}(A)=1$ by showing that if primitive ideals $P$ and $Q$ can be separated by disjoint open sets then they can be separated by a continuous function (Theorem 3.3). The solution of this problem is needed in Section 4 for the proofs of Theorem 4.6 and Corollary 4.7. On the other hand, we show in Example 3.4 that if $\operatorname{Prim}(A)$ is not $\sigma$-compact then it is possible to have $\operatorname{Orc}(A)=1$ and primitive ideals $P$ and $Q$ which can be separated by disjoint open sets but not by continuous functions.

In Section 4 we show that if $A$ is a separable $C_{0}(X)$-algebra then an ideal $J_{x}$ of $A$ which is not locally modular gives rise to an ideal $H_{x}$ of $M(A)$ which is not 2-primal (Theorem 4.2). This leads to Theorem 4.6 which uses local modularity to link $n$-primality of the ideals $J_{x}$ with $n$-primality of the ideals $H_{x}$. From this, the characterization of separable $\mathrm{C}^{*}$-algebras $A$ for which $\operatorname{Orc}(M(A))=1$ follows easily (Corollary 4.7).

If $A$ is a non-unital $C^{*}$-algebra then the multiplier algebra $M(A)$ provides the natural setting for the study of elementary operators. In particular, if $T: A \rightarrow A$ is an elementary operator of the form $T(x)=\sum_{i=1}^{n} a_{i} x b_{i}(x \in A)$, where $a_{i}, b_{i} \in M(A)(1 \leq i \leq n)$ then $T$ extends to $M(A)$, without increase of norm, by using the same formula for $x \in M(A)$. In Section 5 we see how the results of the previous section can be used in estimating the norms of elementary operators on $M(A)$ and in particular the derivation constants $K(M(A))$ and $K_{s}(M(A))$ introduced in [3] (see Section 5 for the definitions). For non-commutative $A$, the optimal value of $K(M(A))$ is $K(M(A))=1 / 2$, which occurs precisely when the norm of every inner derivation of $A$ (or of $M(A)$ ) is equal to twice the distance of the implementing element to the centre of $M(A)$. We show, for example, that if $A$ is a separable non-commutative $C^{*}$-algebra then $K(M(A))=1 / 2$ if and only if every Glimm ideal of $A$ is 3-primal and locally modular (Theorem 5.2). We also give applications to the canonical contraction $\Theta_{Z}: M(A) \otimes_{Z, h} M(A) \rightarrow \mathrm{CB}(A)$ from the central Haagerup tensor product of $M(A)$ to the space of completely bounded linear maps on a $C^{*}$-algebra $A$ (Theorem 5.7 and Theorem 5.9).

We are grateful to the referee for several comments including a suggestion which led to a refinement of our original argument for Lemma 3.1.

\section{A 2-Homogeneous $C^{*}$-Algebra with $\operatorname{Prim}(M(A))$ Non-Hausdorff}

We begin with an example of a 2-homogeneous $\mathrm{C}^{*}$-algebra $A$ for which $\operatorname{Prim}(M(A))$ is nonHausdorff. Recall that a $\mathrm{C}^{*}$-algebra $A$ is $n$-homogeneous if every irreducible representation $A$ has the same finite dimension $n$. If $A$ is $n$-homogeneous then $A$ is quasi-central (i.e. no primitive ideal of $A$ contains the centre of $A$ ) and $\operatorname{Prim}(A)$ is Hausdorff [28, Theorem 
4.2]. Furthermore $M(A)$ is $n$-subhomogeneous (i.e. every irreducible representation is of dimension bounded by $n$ ).

It is known that if $A$ is an $n$-homogeneous $\mathrm{C}^{*}$-algebra then $M(A)$ is $n$-homogeneous if and only if $A$ is of 'finite type' [34, Remark 3.3] (see also [16, 3.16(ii)]). For examples where $A$ is separable and not of finite type, see [39, Corollary 4.8] and [38, Example 3.5]. If $A$ is not of finite type then it is known that $\operatorname{Orc}(M(A))=1$ and that $M(A)$ forms a continuous field of $\mathrm{C}^{*}$-algebras over the Stone-Čech compactification of $\operatorname{Prim}(A)$ [10, Corollary 4.10] (see also the proof of [34, Lemma 3.4]). Some of the fibre algebras corresponding to points in the Stone-Čech remainder are isomorphic to $M_{n}(\mathbb{C})[34$, Lemma 3.4] but little is known about the others; in particular, it appears to be previously unknown whether $\operatorname{Prim}(M(A))$ can be non-Hausdorff (i.e. whether the fibre algebra corresponding to a point in the Stone-Cech remainder can be non-simple).

In this section we exhibit an example with $\operatorname{Prim}(M(A))$ non-Hausdorff. We are grateful to Chuck Akemann, Larry Brown, Bojan Magajna, and Chris Phillips for discussions on this subject.

Theorem 2.1. There exists a 2-homogeneous $C^{*}$-algebra $A$ for which $\operatorname{Prim}(M(A))$ is nonHausdorff.

Proof. For a completely regular Hausdorff space $W$, set $W^{*}=\beta W \backslash W$. Let $\Lambda=\beta \mathbb{R} \backslash \mathbb{N}^{*}$. Then $\Lambda$ is a locally compact Hausdorff space, since $\mathbb{N}^{*}$ is a closed subset of $\beta \mathbb{R}$, but $\Lambda$ is not normal $[23,6 \mathrm{P}]$. To see this, note that since $\Lambda \supseteq \mathbb{R}, \beta \Lambda=\beta \mathbb{R}[23,6.7]$. The closure of $\Lambda \backslash \mathbb{R}$ in $\beta \mathbb{R}$ is equal to $\mathbb{R}^{*}$, and hence $X:=\Lambda \backslash \mathbb{R}$ and $Y:=\mathbb{N}$ are disjoint closed subsets of $\Lambda$ whose closures in $\beta \Lambda=\beta \mathbb{R}$ are not disjoint, since they both contain $\mathbb{N}^{*}$. The space $\Lambda$ is pseudocompact (i.e. every continuous real-valued function on $\Lambda$ is bounded) $[23,6 \mathrm{P}]$. Since $\Lambda$ is locally compact, it follows that $\Lambda \times \Lambda$ is also pseudocompact [43, 8.21]. This implies that $\beta(\Lambda \times \Lambda)=\beta \Lambda \times \beta \Lambda$ by Glicksberg's theorem [43, 8.12]. Note also that, since $\beta \Lambda=\beta \mathbb{R}$, $\Lambda^{*}=\mathbb{N}^{*}$.

Now set $S=X \times Y$ and $T=Y \times X$. Then $S$ and $T$ are homeomorphic disjoint closed subsets of $\Lambda \times \Lambda$, and their closures in $\beta \Lambda \times \beta \Lambda$ both contain $\mathbb{N}^{*} \times \mathbb{N}^{*}$. Let $\Theta$ be the homeomorphism of $\Lambda \times \Lambda$ that takes $(x, y) \in \Lambda \times \Lambda$ to $(y, x)$. Then $\Theta(S)=T$ and $\Theta(T)=S$. Let $B=C_{0}(\Lambda \times \Lambda) \otimes M_{2}(\mathbb{C})$, and let

$$
A=\left\{f \in B: f((x, y))=z f(\Theta((x, y))) z^{*},(x, y) \in S\right\},
$$

where $z$ is the self-adjoint unitary $\operatorname{diag}(1,-1)$. We claim that $A$ is a 2-homogeneous $\mathrm{C}^{*}$ algebra but that $\operatorname{Prim}(M(A))$ is not Hausdorff.

First note that by standard representation theory, every irreducible representation of $A$ is (unitarily equivalent to) a summand of the restriction to $A$ of a point evaluation of $B$. By constructing suitable matrix-valued functions, it can be seen that each such restriction to $A$ is irreducible, mapping $A$ to $M_{2}(\mathbb{C})$. For example, for $(x, y) \in S$, let $U$ and $V$ be disjoint neighbourhoods of $x$ and $y$ respectively in $\Lambda$ and let $g \in C_{0}(\Lambda \times \Lambda)$ such that $g$ is supported in $U \times V$ and $g(x, y)=1$. For $m \in M_{2}(\mathbb{C})$ define a cross-section $f$ by $f(u, v)=$ $g(u, v) m+g(v, u) z^{*} m z((u, v) \in \Lambda \times \Lambda)$. Then $f \in A$ (since $\left.z=z^{*}\right)$ and $f(x, y)=m$. Hence $A$ is 2-homogeneous.

For $(x, y) \in \Lambda \times \Lambda$, let $\pi_{(x, y)}$ denote the irreducible representation of $A$ given by point evaluation at $(x, y)$. Then $\pi_{(x, y)}$ extends to an irreducible representation $\tilde{\pi}_{(x, y)}$ of $M(A)$. 
Hence for $b \in M(A)$, the map $\sigma(b)$ given by $\sigma(b)((x, y))=\tilde{\pi}_{(x, y)}(b)$ defines a bounded $M_{2}(\mathbb{C})$ valued cross-section over $\Lambda \times \Lambda$. By constructing suitable scalar-valued functions $f \in A$ and using the fact that $b f \in A$, it can be checked that $\sigma(b)$ is a continuous cross-section, and satisfies $\sigma(b)((x, y))=z \sigma(b)(\Theta((x, y))) z^{*}$ for $(x, y) \in S$. For example, for $(x, y) \in S$, let $U$, $V$, and $g$ be as above. Define a cross-section $f$ by

$$
f(u, v)=g(u, v) 1+g(v, u) 1 \quad((u, v) \in \Lambda \times \Lambda) .
$$

Then $f \in A$ and $f(u, v)=g(u, v) 1$ for $(u, v) \in U \times V$. Let $\left(u_{\alpha}, v_{\alpha}\right)$ be a net in $\Lambda \times \Lambda$ with $\left(u_{\alpha}, v_{\alpha}\right) \rightarrow(x, y)$. Then eventually $\left(u_{\alpha}, v_{\alpha}\right) \in U \times V$, so

$$
\sigma(b)\left(u_{\alpha}, v_{\alpha}\right) g\left(u_{\alpha}, v_{\alpha}\right)=\sigma(b)\left(u_{\alpha}, v_{\alpha}\right) f\left(u_{\alpha}, v_{\alpha}\right)=(b f)\left(u_{\alpha}, v_{\alpha}\right) \rightarrow(b f)(x, y)=\sigma(b)(x, y) .
$$

Hence $\sigma(b)\left(u_{\alpha}, v_{\alpha}\right) \rightarrow \sigma(b)(x, y)$ because $g\left(u_{\alpha}, v_{\alpha}\right) \rightarrow 1$. Thus $\sigma(b)$ is continuous at $(x, y)$, and

$$
\sigma(b)((x, y))=(b f)((x, y))=z(b f)((y, x)) z^{*}=z \sigma(b)((y, x)) z^{*} .
$$

Since $M(B)$ is the $\mathrm{C}^{*}$-algebra of all bounded continuous functions from $\Lambda \times \Lambda$ into $M_{2}(\mathbb{C})$ [1], the map $\sigma: b \mapsto \sigma(b)$ defines a ${ }^{*}$-homomorphism from $M(A)$ into $M(B)$ fixing elements of $A$; and since $A$ is essential in $M(A)$, we have that $\operatorname{ker} \sigma=\{0\}$. It follows from standard theory that $\sigma(M(A))$ is the idealizer of $A$ in $M(B)$. For $h \in M(B)$, each of the four entry functions has a unique extension to a continuous function on $\beta(\Lambda \times \Lambda)=\beta \Lambda \times \beta \Lambda$, and thus we may consider $M(B)$ as the $\mathrm{C}^{*}$-algebra of continuous $M_{2}(\mathbb{C})$-valued functions on $\beta \Lambda \times \beta \Lambda$.

For $w \in \mathbb{N}^{*}=\Lambda^{*}$, there exist nets $\left(x_{\alpha}\right)$ and $\left(y_{\alpha}\right)$ (both indexed by a neighbourhood base for $w)$ in $X$ and $Y$ respectively such that $x_{\alpha} \rightarrow w$ and $y_{\alpha} \rightarrow w$. Then for $b \in M(A)$ we have $\sigma(b)\left(\left(x_{\alpha}, y_{\alpha}\right)\right) \rightarrow \sigma(b)((w, w))$, and

$$
z^{*} \sigma(b)\left(\left(x_{\alpha}, y_{\alpha}\right)\right) z=\sigma(b)\left(\left(y_{\alpha}, x_{\alpha}\right)\right) \rightarrow \sigma(b)((w, w)) .
$$

Hence $\sigma(b)((w, w))=z^{*} \sigma(b)((w, w)) z$, so $\sigma(b)((w, w))$ is a diagonal matrix. Note that the constant cross-section $d_{1,1}$ given by $d_{1,1}((x, y))=\operatorname{diag}(1,0)((x, y) \in \beta(\Lambda \times \Lambda))$ belongs to the idealizer of $A$ in $M(B)$, and likewise the constant cross-section $d_{2,2}$ given by $d_{2,2}((x, y))=$ $\operatorname{diag}(0,1)$. Hence the map $b \mapsto \sigma(b)((w, w))(b \in M(A))$ is a ${ }^{*}$-homomorphism from $M(A)$

onto $\mathbb{C} \oplus \mathbb{C}$. Thus the net $\left(\tilde{\pi}_{x_{\alpha}, y_{\alpha}}\right)$ converges to two distinct limits in $\widehat{M(A)}$. Finally, $\widehat{M(A)}$ is homeomorphic to $\operatorname{Prim}(M(A))$ since $M(A)$ is 2-subhomogeneous.

The example just given makes heavy use of special properties which can only hold in a non-normal space such as $\Lambda$. This raises the question of whether $\operatorname{Prim}(M(A))$ can be nonHausdorff if $A$ is a $\sigma$-unital $n$-homogeneous $\mathrm{C}^{*}$-algebra (recall that a $C^{*}$-algebra $A$ is $\sigma$-unital if it contains a strictly positive element or, equivalently, a countable approximate unit [37, $3.10 .5])$.

\section{Separation by continuous functions}

In this section we consider the following basic problem (which arises in the proofs of Theorem 4.6 and Corollary 4.7). Let $A$ be a $C^{*}$-algebra and suppose that the relation $\sim$ of Section 1 is an equivalence relation on $\operatorname{Prim}(A)$. Let $P, Q \in \operatorname{Prim}(A)$ with $P \nsim Q$. Does there exist a continuous real function $f$ on $\operatorname{Prim}(A)$ such that $f(P) \neq f(Q)$ ? The second author showed twenty years ago that this is the case if $\operatorname{Prim}(A)$ is compact [40, Corollary 2.7]. Here we use a method recently introduced by Lazar to exhibit such a function if $\operatorname{Prim}(A)$ is 
$\sigma$-compact. On the other hand, we give an example to show that such a function may not exist if $\operatorname{Prim}(A)$ is not $\sigma$-compact.

Following Lazar [30], we work in a more general context. We say that a topological space $X$ is locally compact if every point has a neighbourhood base consisting of compact sets. A subset $F$ of $X$ is a limit set if there is a net in $X$ converging to all the points of $F$. Let $\mathcal{L}(X)$ be the set of closed limit sets of $X$ and set $\mathcal{L}^{\prime}(X)=\mathcal{L}(X) \backslash\{\emptyset\}$. Define a topology $\tau_{s}$ (the Fell topology [22]) on $\mathcal{L}(X)$ as follows. A base for $\tau_{s}$ consists of the family of all sets

$$
U(C, \Phi)=\{F \in \mathcal{L}(X): F \cap C=\emptyset ; F \cap V \neq \emptyset, V \in \Phi\},
$$

where $C$ is a compact subset of $X$ (possibly the empty set) and $\Phi$ is a finite family of open subsets of $X$. Then with this topology $\mathcal{L}(X)$ is a compact Hausdorff space, and $\mathcal{L}^{\prime}(X)$ is a locally compact subspace. If $X$ is $\sigma$-compact then $\mathcal{L}^{\prime}(X)$ is $\sigma$-compact [30, Lemma 2.5].

The first lemma is [15, Vol.2: Exercise 15(b) on p.244]. For completeness, we give a proof in the Appendix.

Lemma 3.1. Let $Y$ be a locally compact, $\sigma$-compact Hausdorff space and let $R$ be an equivalence relation on $Y$. If the graph of $R$ is closed in $Y \times Y$ then $Y / R$ is a normal Hausdorff space.

The proof of the next result is modelled on the methods of Lazar [30].

Proposition 3.2. Let $X$ be a locally compact, $\sigma$-compact space and let $*$ be an equivalence relation on $X$. The following are equivalent:

(i) $X / *$ is a Hausdorff space;

(ii) $X / *$ is a normal Hausdorff space;

(iii) $*$ contains $\sim$ and the graph of $*$ is closed in $X \times X$.

Proof. Let $q: X \rightarrow X / *$ denote the quotient map. Suppose that (i) holds. Let $x, y \in X$ with $x \sim y$ and let $\left(x_{\alpha}\right)$ be a net in $X$ converging to both $x$ and $y$. Then $\left(q\left(x_{\alpha}\right)\right)$ converges to both $q(x)$ and $q(y)$, so $q(x)=q(y)$ since $X / *$ is Hausdorff. Hence $x * y$, so $*$ contains $\sim$. Now let $\left(x_{\alpha}, y_{\alpha}\right)$ be a net in $X \times X$ with limit $(x, y) \in X \times X$ and with $x_{\alpha} * y_{\alpha}$ for each $\alpha$. Then $q\left(x_{\alpha}\right) \rightarrow q(x)$ and $q\left(y_{\alpha}\right) \rightarrow q(y)$. Hence $q(x)=q(y)$, by the Hausdorffness of $X / *$, since $q\left(x_{\alpha}\right)=q\left(y_{\alpha}\right)$ for each $\alpha$. Thus $x * y$, so the graph of $*$ is closed in $X \times X$. This shows that (iii) holds.

Now suppose that (iii) holds. Define a relation $\diamond$ on $\mathcal{L}^{\prime}(X)$ by $F \diamond G$ if there exist $x \in F$ and $y \in G$ such that $x * y$. Then $\diamond$ is reflexive and symmetric. Since $*$ contains $\sim$ we see that $x * x^{\prime}$ for all $x, x^{\prime} \in F$ and hence if $F \diamond G$ then $x^{\prime} * y^{\prime}$ for all $x^{\prime} \in F$ and $y^{\prime} \in G$. Thus $\diamond$ is transitive, and hence we see that $\diamond$ is an equivalence relation on $\mathcal{L}^{\prime}(X)$. We now show that the graph of $\diamond$ is closed. Let $(F, G) \in \mathcal{L}^{\prime}(X) \times \mathcal{L}^{\prime}(X)$ with $(F, G) \notin \diamond$. Let $x \in F$ and $y \in G$. Then $(x, y) \notin *$, and the graph of $*$ is closed, so there exist open neighbourhoods $M$ of $x$ and $N$ of $y$ such that the basic open neighbourhood $M \times N$ of $(x, y)$ does not meet $*$. Set

$$
M^{\prime}=\left\{F^{\prime} \in \mathcal{L}^{\prime}(X): F^{\prime} \cap M \neq \emptyset\right\} \text { and } N^{\prime}=\left\{G^{\prime} \in \mathcal{L}^{\prime}(X): G^{\prime} \cap N \neq \emptyset\right\} .
$$

Then $M^{\prime} \times N^{\prime}$ is an open neighbourhood of $(F, G)$ in $\mathcal{L}^{\prime}(X) \times \mathcal{L}^{\prime}(X)$. If $\left(F^{\prime}, G^{\prime}\right) \in M^{\prime} \times N^{\prime}$ then there exist $x^{\prime} \in F^{\prime} \cap M$ and $y^{\prime} \in G^{\prime} \cap N$. Hence $\left(x^{\prime}, y^{\prime}\right) \in M \times N$ which does not meet $*$. Thus $\left(F^{\prime}, G^{\prime}\right) \notin \diamond$, so the graph of $\diamond$ is closed.

Since $\mathcal{L}^{\prime}(X)$ is $\sigma$-compact [30, Lemma 2.5], it follows from Lemma 3.1 that $\mathcal{L}^{\prime}(X) / \diamond$ is normal and Hausdorff. It remains to show, therefore, that $X / *$ is homeomorphic to $\mathcal{L}^{\prime}(X) / \diamond$. 
Let $Q: \mathcal{L}^{\prime}(X) \rightarrow \mathcal{L}^{\prime}(X) / \diamond$ denote the quotient map, and define a map $\chi: X / * \rightarrow \mathcal{L}^{\prime}(X) / \diamond$ by $\chi(q(x)):=Q(F)$ where $F$ is any closed limit set containing $x$. By definition of $\diamond$, the map $\chi$ is well-defined and injective, and it is trivially surjective. Let $V$ be a closed subset of $\mathcal{L}^{\prime}(X) / \diamond$ and set $W=q^{-1}\left(\chi^{-1}(V)\right)$. Let $\left(x_{\alpha}\right)$ be a net in $W$ with limit $x \in X$. For each $\alpha$ let $F_{\alpha} \in \mathcal{L}^{\prime}(X)$ with $x_{\alpha} \in F_{\alpha}$ and note that $Q\left(F_{\alpha}\right) \in V$ for each $\alpha$. By the $\tau_{s}$-compactness of $\mathcal{L}(X)$, and by passing to a subnet if necessary, we may assume that there exists $F \in \mathcal{L}(X)$ with $F_{\alpha} \rightarrow F$. Then $x \in F$ by [30, Lemma 2.1], so $F \in \mathcal{L}^{\prime}(X)$. It follows that $Q(F) \in V$, since $Q$ is continuous and $V$ is closed. Thus $\chi(q(x)) \in V$, so $x \in W$. This shows that $W$ is a closed subset of $X$ and hence that $\chi^{-1}(V)$ is closed in $X / *$. It follows that the map $\chi$ is continuous.

Now let $V$ be a closed subset of $X / *$ and set $W=q^{-1}(V)$. Let $Y=\left\{F \in \mathcal{L}^{\prime}(X): F \subseteq W\right\}$. Then $Y$ is $\diamond$-saturated since $W$ is $*$-saturated. Let $F \in \mathcal{L}^{\prime}(X) \backslash Y$. Then there exists $x \in F$ such that $x \in U:=X \backslash W$. Hence the set $\left\{G \in \mathcal{L}^{\prime}(X): G \cap U \neq \emptyset\right\}$ is a $\tau_{s}$-neighbourhood of $F$ disjoint from $Y$. It follows that $Y$ is closed and hence that $Q(Y)=\chi(V)$ is closed in $\mathcal{L}^{\prime}(X) / \diamond$. Thus $\chi$ is a homeomorphism and (ii) holds.

Before proceeding, we need to introduce some further terminology. A weaker relation than $\sim$ on $\operatorname{Prim}(A)$ is the relation $\approx$ which is defined by $P \approx Q$ if $P$ and $Q$ cannot be separated by a continuous bounded real-valued function. The relation $\approx$ is always an equivalence relation, and the $\approx$-classes are called Glimm classes. The kernel of a Glimm class (i.e. the intersection of all the primitive ideals in the class) is called a Glimm ideal of $A$, and the set of Glimm ideals is denoted Glimm $(A)$. If $A$ is unital then these are precisely the ideals of $A$ generated by the maximal ideals of the centre of $A$ [24]. The usual topology on $\operatorname{Glimm}(A)$ is the weakest topology such that the functions on $\operatorname{Glimm}(A)$ induced by the continuous bounded functions on $\operatorname{Prim}(A)$ are all continuous [17]. This topology is Hausdorff and completely regular. The map which takes each primitive ideal of $A$ to the unique Glimm ideal which it contains is called the complete regularization map, and is continuous.

A closed two-sided ideal $J$ of a $\mathrm{C}^{*}$-algebra $A$ is said to be primal if whenever $n \geq 2$ and $J_{1}, J_{2}, \ldots, J_{n}$ are ideals of $A$ with product $J_{1} J_{2} \ldots J_{n}=\{0\}$ then at least one of the $J_{i}$ is contained in $J$. This concept arose in [4] where it was shown that a state of $A$ is a weak*limit of factorial states if and only if the kernel of its Gelfand-Naimark-Segal representation is primal. The ideal $J$ is primal if and only if there is a net in $\operatorname{Prim}(A)$ which converges to every point in (a dense subset of) $\operatorname{Prim}(A / J)$ (see [4, Proposition 3.2]). If the variable integer $n$ in the definition of a primal ideal is replaced by a fixed integer $n \geq 2$, we obtain the notion of an $n$-primal ideal $J$. By [8, Lemma 1.3], the ideal $J$ is $n$-primal if and only if the ideal $\bigcap_{i=1}^{n} P_{i}$ is primal whenever $P_{1}, \ldots, P_{n}$ are primitive ideals of A containing $J$.

If an ideal $J$ is 2-primal then $P \sim Q$ whenever $P$ and $Q$ are primitive ideals containing $J$. Hence $P$ and $Q$ lie in the same Glimm class. It follows that every 2-primal ideal contains a unique Glimm ideal. The condition that every Glimm ideal be 2-primal is equivalent to requiring that for $P, Q \in \operatorname{Prim}(A), P \sim Q$ if and only if $P \approx Q$. Evidently this condition implies that $\sim$ is an equivalence relation on $\operatorname{Prim}(A)$, i.e. that $\operatorname{Orc}(A)=1$, and the question naturally arises as to whether the reverse implication holds. It is already known that this is so when $A$ is unital [40, Corollary 2.7], and we are now able to extend this to the case when $A$ is $\sigma$-unital.

Theorem 3.3. Let $A$ be a $\sigma$-unital $C^{*}$-algebra. 
(a) The relation $\approx$ is the smallest equivalence relation on $\operatorname{Prim}(A)$ which contains $\sim$ and which has closed graph in $\operatorname{Prim}(A) \times \operatorname{Prim}(A)$.

(b) Define a relation $*$ on $\operatorname{Prim}(A)$ by $P * Q$ if $P$ and $Q$ belong to the same $\sim$-component. Then the graph of $*$ is closed in $\operatorname{Prim}(A) \times \operatorname{Prim}(A)$ if and only if every Glimm class is a $\sim$-component of $\operatorname{Prim}(A)$.

(c) If $\operatorname{Orc}(A)=1$ then $\sim$ and $\approx$ coincide on $\operatorname{Prim}(A)$ and every Glimm ideal of $A$ is 2-primal.

Proof. (a) Let $\mathcal{R}$ be the set of all equivalence relations on $\operatorname{Prim}(A)$ which contain $\sim$ and which have closed graph. Clearly the equivalence relation $\approx$ contains $\sim$, and we now show that the graph of $\approx$ is closed. Let $\left(P_{\alpha}, Q_{\alpha}\right)$ be a net in $\operatorname{Prim}(A) \times \operatorname{Prim}(A)$ with $\operatorname{limit}$ $(P, Q) \in \operatorname{Prim}(A) \times \operatorname{Prim}(A)$. If $P \not \approx Q$ then there is a continuous bounded real-valued function $f$ on $\operatorname{Prim}(A)$ such that $f(P) \neq f(Q)$. Hence eventually $f\left(P_{\alpha}\right) \neq f\left(Q_{\alpha}\right)$. It follows that the graph of $\approx$ is closed, and hence that $\approx$ belongs to $\mathcal{R}$. Now let $R=\bigcap\{S: S \in \mathcal{R}\}$. Then $R$ is a equivalence relation on $\operatorname{Prim}(A)$ containing $\sim$ and with closed graph. Thus $R$ is contained in $\approx$. On the other hand, since $A$ is $\sigma$-unital, $\operatorname{Prim}(A)$ is $\sigma$-compact and so, by Proposition 3.2, $\operatorname{Prim}(A) / R$ is a normal Hausdorff space and hence is completely regular. This implies that $R$ contains $\approx$, and thus $R$ coincides with $\approx$.

(b) Clearly $*$ is the smallest equivalence relation on $\operatorname{Prim}(A)$ containing $\sim$. Hence by part (a), $*$ coincides with $\approx$ if and only if the graph of $*$ is closed. On the other hand $*$ coincides with $\approx$ if and only if every $\approx$-class is a $\sim$-component of $\operatorname{Prim}(A)$.

(c) Let $\left(P_{\alpha}, Q_{\alpha}\right)$ be a net in $\operatorname{Prim}(A) \times \operatorname{Prim}(A)$ with $\operatorname{limit}(P, Q) \in \operatorname{Prim}(A) \times \operatorname{Prim}(A)$. If $P \nsim Q$ then eventually $P_{\alpha} \nsim Q_{\alpha}$. Thus the graph of $\sim$ is always closed in $\operatorname{Prim}(A) \times \operatorname{Prim}(A)$. If $\operatorname{Orc}(A)=1$ then $\sim$ itself is an equivalence relation with closed graph, so $\sim$ and $\approx$ coincide by part (a). Hence every Glimm ideal of $A$ is 2-primal.

Since the relations $\sim$ and $\approx$ can be defined on any topological space, the proof of Theorem 3.3 may be applied to any locally compact, $\sigma$-compact space $X$ instead of $\operatorname{Prim}(A)$, with the analogous conclusions.

One application of Theorem 3.3 is to the following problem. For $N \geq 3$, let $G_{N}$ be the 'threadlike' nilpotent Lie group of dimension $N$ [8] (so that $G_{3}$ is the continuous Heisenberg group). It was shown in [8, Corollary 3.10] that $\sim$ is an equivalence relation on $\operatorname{Prim}(A)$ for the group $\mathrm{C}^{*}$-algebra $A=C^{*}\left(G_{N}\right)$ except when $N \equiv 0(\bmod 4)$ with $N>4$. If $N$ is odd then $\sim$ and $\approx$ coincide on $\operatorname{Prim}(A)$ [8, Corollary 3.7 and remark after Corollary 3.10], but the relation $\approx$ was left undetermined in the case $N \equiv 2(\bmod 4)($ see $[14, \mathrm{p} .1426])$. It follows from Theorem 3.3(c) that $\sim$ and $\approx$ must coincide in this case.

It is worth remarking that group $\mathrm{C}^{*}$-algebras with $\operatorname{Orc}(A)=1$ are surprisingly common. For example, in addition to the 'threadlike' nilpotent Lie groups $G_{N}$ just mentioned, $A=C^{*}(G)$ has this property if $G$ is any of the following groups: $S L(2, \mathbb{C}$ ) (see [20] and [19, 18.9.13]); an amenable [SIN] group [27]; a universal simply connected, 2-step nilpotent Lie group $W_{n}(n \geq 2)$ [8, Corollary 2.8]; any simply connected, nilpotent Lie group of dimension not exceeding 6 . For this last claim, note that this class contains thirty-two indecomposable non-abelian groups, listed in [35]. All but $G_{5,2}, G_{5,4}$ and $G_{6,18}$ were observed to have $\operatorname{Orc}\left(C^{*}(G)\right)=1$ in [5] or [8]. The orbit data in [35] is sufficient to determine the relation $\sim$ in the three remaining cases, from which it follows that $\operatorname{Orc}\left(C^{*}(G)\right)=1$ for these too. Finally, if $G$ is a Type I group and $H$ is any other locally compact group then $C^{*}(G \times H)$ is isomorphic to the unique $C^{*}$-tensor product $C^{*}(G) \otimes C^{*}(H)$ and has primitive ideal space 
canonically homeomorphic to $\operatorname{Prim}\left(C^{*}(G)\right) \times \operatorname{Prim}\left(C^{*}(H)\right)$. It follows easily from this that $\operatorname{Orc}\left(C^{*}(G \times H)\right)=1$ if and only if $\operatorname{Orc}\left(C^{*}(G)\right)=1=\operatorname{Orc}\left(C^{*}(H)\right)$ (cf. [26, Lemmas 3.1 and $3.2])$.

We close this section with an example where $\operatorname{Orc}(A)=1$ but $\sim$ and $\approx$ do not coincide.

Example 3.4. $A$ (non- $\sigma$-unital) $C^{*}$-algebra $A$ for which $\sim$ is an equivalence relation (i.e. for which $\operatorname{Orc}(A)=1$ ) but for which $\sim$ is not equal to $\approx$.

Let $X$ be a non-normal locally compact Hausdorff space with disjoint closed subsets $Y$ and $Z$ which cannot be completely separated, i.e. such that there is no continuous function on $X$ taking the value 1 on $Y$ and 0 on $Z$. Set $B=C_{0}(X)$, and let $C$ (respectively $D$ ) be the *-homomorphic image of $B$ in $C^{b}(Y)$ (respectively $C^{b}(Z)$ ) obtained by restricting functions in $B$ to $Y$ (respectively $Z$ ). By [9, Theorem 3.6] there are quasi-standard $\mathrm{C}^{*}$-algebras $E$ and $F$ with Glimm ideals $G$ and $H$ respectively such that $C \cong E / G$ and $D \cong F / H$. Let $A_{1}$ be the direct sum of $B, E$ and $F$, and let $A$ be the $\mathrm{C}^{*}$-subalgebra of $A_{1}$ consisting of those elements $(b, e, f) \in A_{1}$ such that $\left.b\right|_{Y}=e+G$ in $C$ and $\left.b\right|_{Z}=f+H$ in $D$.

Then $\sim$ is an equivalence relation on $\operatorname{Prim}(A)$ with the primitive ideals associated with $Y$ forming one $\sim$-class, and the primitive ideals associated with $Z$ forming another. But there is no continuous bounded function on $\operatorname{Prim}(A)$ separating these two $\sim$-classes, and therefore together they form a single Glimm class. Hence $\operatorname{Orc}(A)=1$ but $\sim$ and $\approx$ do not coincide on $\operatorname{Prim}(A)$. Note that the graph of $\sim$ is automatically closed by the proof of Theorem 3.3(c), and hence this example shows that parts (a), (b), and (c) of Theorem 3.3 can all fail in the non- $\sigma$-unital case.

\section{Characterizing $\operatorname{Orc}(M(A))=1$}

In this section we prove the central result of the paper which is to give an intrinsic characterization of the separable $\mathrm{C}^{*}$-algebras $A$ for which $\operatorname{Orc}(M(A))=1$, or equivalently, for which every Glimm ideal of $M(A)$ is 2-primal.

For a proper, closed, two-sided ideal $J$ in a $\mathrm{C}^{*}$-algebra $A$, let $\tilde{J}$ denote the strict closure of $J$ in $M(A)$ (see [10, Proposition 1.1]). Note that for, $P \in \operatorname{Prim}(A), \tilde{P}$ is the unique primitive ideal of $M(A)$ such that $\tilde{P} \cap A=P$. We begin with a lemma relating the primality of $J$ to that of $\tilde{J}$.

Lemma 4.1. Let $A$ be a $C^{*}$-algebra and let $J$ be a closed, two-sided ideal in $A$ with strict closure $\tilde{J}$ in $M(A)$. Then for $n \geq 2, J$ is n-primal (respectively primal) in $A$ if and only if $\tilde{J}$ is n-primal (respectively primal) in $M(A)$.

Proof. Since an ideal is primal if and only if it is $n$-primal for all $n \geq 2$, it is enough to prove the result for $n$-primality. If $J$ is $n$-primal then $\tilde{J}$ is $n$-primal by [10, Lemma 4.5] and its proof.

Conversely, suppose that $\tilde{J}$ is $n$-primal. Let $P_{1}, \ldots, P_{n} \in \operatorname{Prim}(A / J)$. Then by the $n$ primality of $\tilde{J}$ there is a net in $\operatorname{Prim}(M(A))$ converging to $\tilde{P}_{1}, \ldots, \tilde{P}_{n}$, and by the density of $\operatorname{Prim}(A)^{\sim}$ in $\operatorname{Prim}(M(A))$ this net may be chosen to be of the form $\left(\tilde{P}_{\alpha}\right)$ where each $P_{\alpha} \in \operatorname{Prim}(A)$. Hence $\left(P_{\alpha}\right)$ converges to $P_{1}, \ldots, P_{n}$, so $J$ is $n$-primal.

Next we give the main technical result of this section. It is convenient to work in the context of $C_{0}(X)$-algebras. Recall that a $\mathrm{C}^{*}$-algebra $A$ is a $C_{0}(X)$-algebra if there is a continuous map $\phi$ from $\operatorname{Prim}(A)$ to the locally compact Hausdorff space $X$. The map $\phi$ is 
called the base map and the image of $\phi$ is denoted $X_{\phi}$. For $x \in X_{\phi}$, we define $H(x):=$ $\phi^{-1}(x)$ and $J_{x}:=\operatorname{ker}(H(x))$. The map $\phi$ has a unique extension to a continuous map $\bar{\phi}: \operatorname{Prim}(M(A)) \rightarrow \beta X$, such that $\bar{\phi}(\tilde{P})=\phi(P)$ for all $P \in \operatorname{Prim}(A)$; and for $x \in X_{\phi}$ we define $H_{x}:=\operatorname{ker}\left((\bar{\phi})^{-1}(x)\right)$. Then $J_{x} \subseteq H_{x} \subseteq \tilde{J}_{x}$, so $H_{x}$ is strictly closed if and only if $H_{x}=\tilde{J}_{x}$. When $H_{x}=\tilde{J}_{x}$ we say that spectral synthesis holds at $x$ [12]. For further details, see [10] and [12].

Theorem 4.2. Let $A$ be a separable $C_{0}(X)$-algebra with base map $\phi$ and let $x \in X_{\phi}$ with $H_{x}$ not strictly closed. Then $H_{x}$ is not 2-primal.

Proof. Let $b \in \tilde{J}_{x}$ such that $\left\|b+H_{x}\right\|=1$, and let $Q$ be a primitive ideal of $M(A)$ containing $H_{x}$ such that $\|b+Q\|>1 / 2$. Set $U=\{S \in \operatorname{Prim}(M(A)):\|b+S\|>1 / 2\}$, an open neighbourhood of $Q$ in $\operatorname{Prim}(M(A))$, and set $V=\{P \in \operatorname{Prim}(A): \tilde{P} \in U\}$. Since $\operatorname{Prim}(A)^{\sim}$ is a dense open subset of $\operatorname{Prim}(M(A)), V$ is open in $\operatorname{Prim}(A)$ and $\tilde{V}$ is dense in $U$. Let $T \in H(x)$. If $\tilde{T} \nsim Q$ then $H_{x}$ is not 2-primal, so we may assume that $\tilde{T} \sim Q$. Then $\tilde{T}$ lies in the closure of $U$ in $\operatorname{Prim}(M(A))$ and hence in the closure of $\tilde{V}$. Thus $T$ lies in the closure of $V$ in $\operatorname{Prim}(A)$. Since $A$ is separable, $T$ has a countable neighbourhood base in $\operatorname{Prim}(A)$ $[19,3.3 .4]$ and so there exists a sequence $\left(P_{n}\right)$ in $V$ such that $P_{n} \rightarrow T$.

Let $I_{b}$ be the closed ideal of $A$ generated by $b$, that is, the norm-closure of $A b A$. Using an approximate identity for $A$, we obtain that $a b, b a \in I_{b}(a \in A)$ and hence $b \in \tilde{I}_{b}$. Set $W=\phi\left(\operatorname{Prim}\left(I_{b}\right)\right)$ (where $\operatorname{Prim}\left(I_{b}\right)$ is regarded as a subset of $\operatorname{Prim}(A)$ in the usual way). If $P \in \operatorname{Prim}(A)$ and $P \supseteq I_{b}$ then $b \in \tilde{I}_{b} \subseteq \tilde{P}$ and so $P \notin V$. It follows that $V \subseteq \operatorname{Prim}\left(I_{b}\right)$ and hence $\phi(V) \subseteq W$. On the other hand, if $P \in H(x)$ then $b \in \tilde{J}_{x} \subseteq \tilde{P}$ and so $I_{b} \subseteq \tilde{P} \cap A=P$. Thus $x \notin W$. Since $A$ is separable, $X_{\phi}$ is perfectly normal [12, Lemma 3.9] and so there is a continuous function $\rho: X_{\phi} \rightarrow[0, \infty)$ with zero set equal to the closed set $\{x\}$. In particular, $\rho(y)>0$ for all $y \in W$.

We shall use the following observation. Let $f$ be any continuous bounded function on $W$. Then $f \circ \phi$ defines a continuous bounded function on $\operatorname{Prim}\left(I_{b}\right)$, and hence defines a unique central multiplier $z_{f}$ of $I_{b}$ such that

$$
z_{f} c+\left(P \cap I_{b}\right)=f(\phi(P))\left(c+\left(P \cap I_{b}\right)\right)
$$

for all $c \in I_{b}$ and $P \in \operatorname{Prim}(A)$ such that $P \nsupseteq I_{b}$. We now define a double centralizer $(R, L)$ on $A$ by $R(a)=z_{f}(a b), L(a)=z_{f}(b a)(a \in A)$. Since $I_{b}=I_{b}^{2},\left(z_{f} c\right) a=z_{f}(c a)$ and $a\left(c z_{f}\right)=(a c) z_{f}$ for all $a \in A$ and $c \in I_{b}$. Thus, for $a_{1}, a_{2} \in A$,

$$
R\left(a_{1}\right) a_{2}=z_{f}\left(a_{1} b a_{2}\right)=\left(a_{1} b a_{2}\right) z_{f}=a_{1} L\left(a_{2}\right) .
$$

Hence by $[37,3.12 .3]$ there exists $b_{f} \in M(A)$ such that $b_{f} a=z_{f}(b a)$ and $a b_{f}=z_{f}(a b)$ for all $a \in A$. Let $y \in W, a \in A$ and $P \in \operatorname{Prim}(A)$ with $P \supseteq J_{y}$. If $I_{b} \not \subset P$ then

$$
b_{f} a-f(y) b a=z_{f}(b a)-f(y) b a \in P \cap I_{b} \subseteq P .
$$

On the other hand, if $I_{b} \subseteq P$ then $b_{f} a-f(y) b a=z_{f}(b a)-f(y) b a \in I_{b} \subseteq P$. Thus in either case, $b_{f} a-f(y) b a \in P$. Since this is true for all such $P, b_{f} a-f(y) b a \in J_{y}$ and similarly $a b_{f}-f(y) a b \in J_{y}$. Hence $b_{f}-f(y) b \in \tilde{J}_{y}$.

Recall that $P_{n} \in V$ and set $x_{n}=\phi\left(P_{n}\right)$ so that $x_{n} \in \phi(V) \subseteq W$ and

$$
\left\|b+\tilde{J}_{x_{n}}\right\| \geq\left\|b+\tilde{P}_{n}\right\|>1 / 2 \quad(n \geq 1) .
$$


Since $\phi$ is continuous, $x_{n} \rightarrow x$ and hence $\rho\left(x_{n}\right) \rightarrow 0$. By passing to a subsequence, we may suppose that $\rho\left(x_{n}\right)$ is strictly decreasing to zero. Let $g:(0, \infty) \mapsto(0, \infty)$ be a continuous function such that $g\left(\rho\left(x_{n}\right)\right)=\frac{2}{\pi(2 n-1)}(n \geq 1)$. Let $f$ be the continuous bounded function on $W$ defined by

$$
f(y)=\sin \left(\frac{1}{g(\rho(y))}\right)
$$

$(y \in W)$. Then $f\left(x_{2 m}\right)=-1$ and $f\left(x_{2 m-1}\right)=1(m \geq 1)$. Let $f^{+}$and $f^{-}$be the positive and negative parts of $f$. Then $f^{+}$and $f^{-}$are non-zero continuous bounded functions on $W$ and $f^{+} f^{-}=0$. Set $b_{1}=b_{f^{+}}$and $b_{2}=b_{f^{-}}$. Then $b_{1} A b_{2}=\{0\}$. For $c \in M(A)$ and $a \in A, b_{1} c b_{2} a=\lim b_{1} c u_{\lambda} b_{2} a=0$ (where $\left(u_{\lambda}\right)$ is an approximate identity for $A$ ) and similarly $a b_{1} c b_{2}=0$. Thus $b_{1} c b_{2}=0$ and so $b_{1} M(A) b_{2}=\{0\}$. Hence $J_{1} J_{2}=\{0\}$ where $J_{1}$ and $J_{2}$ are the smallest closed ideals in $M(A)$ containing $b_{1}$ and $b_{2}$ respectively. On the other hand, since $b_{1}-f^{+}\left(x_{2 n-1}\right) b \in \tilde{J}_{2 n-1}$, we have

$$
\left\|b_{1}+H_{x_{2 n-1}}\right\| \geq\left\|b_{1}+\tilde{J}_{x_{2 n-1}}\right\|=\left|\left(f^{+}\right)\left(x_{2 n-1}\right)\right|\left\|b+\tilde{J}_{x_{2 n-1}}\right\|>1 / 2
$$

for all $n$, and similarly $\left\|b_{2}+H_{x_{2 n}}\right\|>1 / 2$ for all $n$. Hence neither $b_{1}$ nor $b_{2}$ belong to $H_{x}$, by the upper semi-continuity of norm functions on $X$. Thus $H_{x}$ is not 2-primal.

If $\phi$ is the complete regularization map for $\operatorname{Prim}(A)$ then $H_{x}$ is a Glimm ideal of $M(A)$ (see the remarks after Proposition 4.4). Thus we see that if $A$ is separable then necessary conditions for $\operatorname{Orc}(M(A))=1$ are that $\operatorname{Orc}(A)=1$ and that every Glimm ideal of $M(A)$ which does not contain $A$ should be strictly closed. This latter property has been called 'global spectral synthesis' in [12].

We give two examples which illustrate Theorem 4.2. The second of these shows that when $A$ is non-separable it is possible for $H_{x}$ to be 2-primal but not strictly closed.

Example 4.3. (i) Let $A$ be the $\mathrm{C}^{*}$-algebra of sequences $x=\left(x_{n}\right)$ of $2 \times 2$ complex matrices such that $x_{n} \rightarrow \operatorname{diag}(\lambda(x), 0)$ as $n \rightarrow \infty$. Then $M(A)$ is the $\mathrm{C}^{*}$-algebra of sequences $y=\left(y_{n}\right)$ of matrices whose off-diagonal terms converge to zero, whose $(1,1)$-entries converge to a limit $\tilde{\lambda}(y)$, and whose $(2,2)$-entries form a bounded sequence. Set $J_{\infty}=\operatorname{ker} \lambda$, and for $n \geq 1$, set $J_{n}=\left\{x \in A: x_{n}=0\right\}$. It is well-known that $\operatorname{Prim}(A)=\left\{J_{n}: n \geq 1\right\} \cup\left\{J_{\infty}\right\}$ and is homeomorphic to the Hausdorff space $\mathbb{N} \cup\{\infty\}$. Hence Prim $(A)=\operatorname{Glimm}(A)$. It is not difficult to see that $\tilde{J}_{\infty}=\operatorname{ker} \tilde{\lambda}$ while

$$
H_{\infty}=\left\{y \in M(A): y_{n} \rightarrow 0\right\}=J_{\infty} .
$$

Hence $H_{\infty}$ is not strictly closed (see also [12, Proposition 2.6(iii)]) so it follows from Theorem 4.2 that $H_{\infty}$ is not 2-primal. This can also be seen directly by considering, for instance, the ideals of elements in $M(A)$ whose even (respectively, odd) terms are zero.

(ii) Let $X=\beta \mathbb{N}$ and let $y \in \beta \mathbb{N} \backslash \mathbb{N}$. Let $A$ be the $\mathrm{C}^{*}$-algebra of continuous functions $f$ from $X$ into the $2 \times 2$ complex matrices such that $f(y)=\operatorname{diag}(\lambda(f), 0)$. Routine arguments show that $M(A)$ is the algebra of all continuous functions $f$ from $X$ into the $2 \times 2$ complex matrices such that $f(y)=\operatorname{diag}(\lambda(f), \mu(f))$ (cf. [13, Example 12]). Then, as in example (i), $\operatorname{Prim}(A)$ is homeomorphic to the base-space $\beta \mathbb{N}$ and every primitive ideal of $A$ is a Glimm ideal. Set $J_{y}=\{f \in A: \lambda(f)=0\}$. Then $\tilde{J}_{y}=\{f \in M(A): \lambda(f)=0\}$ while $H_{y}=\{f \in M(A): \lambda(f)=\mu(f)=0\}$. Hence $H_{y}$ is not strictly closed. Theorem 4.2 does not apply, since $A$ is non-separable, and in fact $H_{y}$ is primal. 
We now need some further definitions [12]. Let $A$ be a $C_{0}(X)$-algebra with base map $\phi: \operatorname{Prim}(A) \rightarrow X$. For $x \in X_{\phi}$ we say that $\phi$ is locally closed at $x[29, \S 13$.XIV] if whenever $Y$ is a closed subset of $\operatorname{Prim}(A)$ such that $x$ lies in the closure of $\phi(Y)$ then $x \in \phi(Y)$, that is, $Y \cap H(x)$ is non-empty.

For the next definition, it is helpful to have the following notation. For $x \in X_{\phi}$, let $\partial H(x)$ be the boundary and $U(x)$ the interior of $H(x)$ in $\operatorname{Prim}(A)$. We say that $J_{x}$ is locally modular if for each $P \in \partial H(x)$ there exists a relatively open neighbourhood $V$ of $P$ in $\operatorname{Prim}(A) \backslash U(x)$ such that $A / \operatorname{ker} V$ is a unital $\mathrm{C}^{*}$-algebra. Examples of locally modular ideals are given in [12, Section 3 and Examples 6.4].

For a useful formulation, which is equivalent to local modularity when $A$ is $\sigma$-unital, we need a slight variant of the definition of $\sim$. For $Q, R \in \operatorname{Prim}(M(A)) \backslash \tilde{U}(x)$ we say that $Q \sim_{x} R$ if there is a net $\left(P_{\alpha}\right)$ in $\operatorname{Prim}(A) \backslash U(x)$ such that $\left(\tilde{P}_{\alpha}\right)$ converges to both $Q$ and $R$. If $A$ is $\sigma$-unital then $J_{x}$ is locally modular if and only if for all $P \in \partial H(x)$ and $R \in \operatorname{Prim}(M(A) / A), \tilde{P} \chi_{x} R[12$, Lemma 3.3].

Proposition 4.4. Let $A$ be a separable $C_{0}(X)$-algebra with base map $\phi$. Let $x \in X_{\phi}$ and let $n \geq 2$. Then the following are equivalent:

(a) $H_{x}$ is n-primal (respectively, primal);

(b) $J_{x}$ is n-primal (respectively, primal) and $H_{x}$ is strictly closed;

(c) $J_{x}$ is n-primal (respectively, primal) and locally modular, and $\phi$ is locally closed at $x$.

Proof. (a) $\Rightarrow$ (b) If $H_{x}$ is $n$-primal then so too is the containing ideal $\tilde{J}_{x}$, and then $J_{x}$ is $n$-primal by Lemma 4.1. Furthermore $H_{x}$ must be strictly closed by Theorem 4.2.

(b) $\Rightarrow$ (a) This follows from Lemma 4.1 since $\tilde{J}_{x}$ is the strict closure of $H_{x}$.

(b) $\Leftrightarrow(\mathrm{c})$ This follows from [12, Corollary 3.10].

Any $C^{*}$-algebra $A$ may be viewed as a $C_{0}(X)$-algebra where the base map $\phi$ is the complete regularization map and where $X$ is either $\operatorname{Glimm}(A)$ (if this is locally compact) or the Stone-Čech compactification of $\operatorname{Glimm}(A)$. In either case $X_{\phi}=\operatorname{Glimm}(A)$. From now on, for the sake of generality and definiteness, we will take $X=\beta \operatorname{Glimm}(A)$ as the target of the complete regularization map for $A$. As noted in [6, p. 2054] (with slightly different notation), if $G \in X_{\phi}=\operatorname{Glimm}(A)$ then $J_{G}=G$ and $H_{G}=\iota(G)$, where $\iota: \beta \operatorname{Glimm}(A) \rightarrow$ $\operatorname{Glimm}(M(A))$ is the canonical homeomorphism described in [10, Proposition 4.7]. There is a continuous map $\bar{\phi}: \operatorname{Prim}(M(A)) \rightarrow X=\beta \operatorname{Glimm}(A)$ such that $\bar{\phi}(\tilde{P})=\phi(P)$ for all $P \in \operatorname{Prim}(A)$ and $\iota \circ \bar{\phi}=\phi_{M(A)}$, where $\phi_{M(A)}: \operatorname{Prim}(M(A)) \rightarrow \operatorname{Glimm}(M(A))$ is the complete regularization map for $M(A)\left[6\right.$, p. 2054]. For $x \in X=\beta \operatorname{Glimm}(A), H_{x}=$ $\bigcap\{Q \in \operatorname{Prim}(A): \bar{\phi}(Q)=x\}$ by definition. But $\bar{\phi}(Q)=x$ if and only if $\phi_{M(A)}(Q)=\iota(x)$ and so $H_{x}=\iota(x) \in \operatorname{Glimm}(M(A))$. Since $\iota$ is surjective,

$$
\operatorname{Glimm}(M(A))=\left\{H_{x}: x \in \beta \operatorname{Glimm}(A)\right\} .
$$

The topology on $X_{\phi}=\operatorname{Glimm}(A)$ is the complete regularization topology, and this coincides with the quotient topology induced from $\operatorname{Prim}(A)$ by $\phi$ if $A$ is $\sigma$-unital [30, Theorem $2.6]$.

Proposition 4.5. Let $A$ be a $\sigma$-unital $C_{0}(X)$-algebra where $\phi$ is the complete regularization map for $\operatorname{Prim}(A)$ and suppose that $\operatorname{Orc}(A)=1$. Suppose that the Glimm ideal $J_{x}$ is locally modular for all $x \in X_{\phi}$. Then, for all $x \in X_{\phi}$, $\phi$ is locally closed at $x$ and $H_{x}$ is strictly closed. 
Proof. The main thing is to show that $\phi$ is locally closed at $x$ for all $x \in X_{\phi}$. As a matter of fact, this follows from [12, Theorem 5.3], but the proof of that theorem is somewhat involved and we can give a much simpler proof in the present case where $\operatorname{Orc}(A)=1$ by exploiting the new result in Theorem 3.3(c).

Let $Y$ be a closed subset of $\operatorname{Prim}(A)$. We are required to show that $\phi(Y)$ is closed in $X_{\phi}$. But $A$ is $\sigma$-unital and so it is enough to show that $\phi^{-1}(\phi(Y))$ is closed [30, Theorem 2.6]. Since $\sim$ and $\approx$ coincide on $\operatorname{Prim}(A)$ (Theorem 3.3(c)), this amounts to showing that $Y^{1}:=\{Q \in \operatorname{Prim}(A): \exists P \in Y$ with $P \sim Q\}$ is closed.

Let $\left(Q_{\alpha}\right)$ be a net in $Y^{1}$ with $Q_{\alpha} \rightarrow Q \in \operatorname{Prim}(A)$. Set $x=\phi(Q)$. Suppose, for a contradiction, that $Q \notin Y^{1}$. For each $\alpha$ there exists $P_{\alpha} \in Y$ such that $P_{\alpha} \sim Q_{\alpha}$. By the compactness of $\operatorname{Prim}(M(A))$, and by passing to a subnet if necessary, we may assume that $\tilde{P}_{\alpha} \rightarrow R$ for some $R \in \operatorname{Prim}(M(A))$. Then $R \sim \tilde{Q}$. If $R \in \tilde{Y}$ then $Q \in Y^{1}$, contrary to our assumption. Hence $R \supseteq A$, since $Y$ is closed. If $Q_{\alpha} \in H(x)$ for any $\alpha$ then $P_{\alpha} \in H(x)$ since $P_{\alpha} \sim Q_{\alpha}$. This implies that $H(x) \subseteq Y^{1}$, since $\sim$ and $\approx$ coincide, and hence that $Q \in H(x) \subseteq Y^{1}$, contrary to our assumption. Thus we see that no $Q_{\alpha}$ belongs to $H(x)$. It follows that $Q$ lies in the boundary of $H(x)$.

Let $U$ and $V$ be open neighbourhoods of $\tilde{Q}$ and of $R$ respectively in $\operatorname{Prim}(M(A))$. Set $U^{\prime}=\{P \in \operatorname{Prim}(A): \tilde{P} \in U\}$ and $V^{\prime}=\{P \in \operatorname{Prim}(A): \tilde{P} \in V\}$. Then there exists $\alpha$ such that $Q_{\alpha} \in U^{\prime}$ and $P_{\alpha} \in V^{\prime}$. Since $Q_{\alpha}, P_{\alpha} \notin H(x), U^{\prime \prime}=U^{\prime} \backslash H(x)$ and $V^{\prime \prime}=V^{\prime} \backslash H(x)$ are open neighbourhoods of $Q_{\alpha}$ and $P_{\alpha}$ respectively. Since $Q_{\alpha} \sim P_{\alpha}$, there exists $P \in \operatorname{Prim}(A)$ such $P \in U^{\prime \prime} \cap V^{\prime \prime}$. Hence $P \notin H(x)$ and $\tilde{P} \in U \cap V$. It follows that $\tilde{Q} \sim_{x} R$. By [12, Lemma 3.3], this contradicts the local modularity hypothesis on $J_{x}$. Thus $Q \in Y^{1}$ and $Y^{1}$ is closed. It follows, as above, that $\phi$ is locally closed at each $x \in X_{\phi}$. Hence, using the local modularity of $J_{x}$ again, $H_{x}$ is strictly closed for all $x \in X_{\phi}$ [12, Proposition 3.4].

Theorem 4.6. Let $A$ be a separable $C^{*}$-algebra and let $\phi$ be the complete regularization map for $\operatorname{Prim}(A)$. Then for $n \geq 2, H_{x}$ is n-primal (respectively, primal) for all $x \in \beta X_{\phi}$ if and only if $J_{x}$ is locally modular and n-primal (respectively, primal) for all $x \in X_{\phi}$.

Proof. Suppose first that $H_{x}$ is $n$-primal (respectively primal) for all $x \in \beta X_{\phi}$. Then it follows from Proposition 4.4 that $J_{x}$ is locally modular and $n$-primal (respectively primal) for all $x \in X_{\phi}$.

For the converse, it suffices to assume that $A$ is $\sigma$-unital rather than separable. Suppose that $J_{x}$ is locally modular and $n$-primal (respectively primal) for all $x \in X_{\phi}$. In particular, this implies that $\operatorname{Orc}(A)=1$. By Proposition $4.5, H_{x}$ is strictly closed for all $x \in X_{\phi}$. Hence $H_{x}=\tilde{J}_{x}$ is $n$-primal (respectively primal) for all $x \in X_{\phi}=\operatorname{Glimm}(A)$ by Lemma 4.1. Since $A$ is $\sigma$-unital, $X_{\phi}$ is normal [11, p. 366]. Recalling that $\operatorname{Glimm}(M(A))=\left\{H_{x}: x \in\right.$ $\beta \operatorname{Glimm}(A)\}$, we obtain from [6, Corollary 3.3] that $H_{x}$ is $n$-primal (respectively primal) for all $x \in \beta X_{\phi}$.

Translating Theorem 4.6 back into the language of Glimm ideals, we obtain our main result. We observe that condition (b) below is intrinsic to $A$.

Corollary 4.7. Let $A$ be a separable $C^{*}$-algebra. Then the following are equivalent:

(a) $\operatorname{Orc}(M(A))=1$;

(b) $\operatorname{Orc}(A)=1$ and every Glimm ideal of $A$ is locally modular;

(c) $\operatorname{Orc}(A)=1$ and $H_{G}$ is strictly closed in $M(A)$ for all $G \in \operatorname{Glimm}(A)$. 
Proof. Let $\phi$ be the complete regularization map for $\operatorname{Prim}(A)$ so that $X_{\phi}=\operatorname{Glimm}(A)$ and the ideals $J_{x}\left(x \in X_{\phi}\right)$ are the Glimm ideals of $A$.

(a) $\Rightarrow(\mathrm{b})$. Suppose that $\operatorname{Orc}(M(A))=1$. Then $\operatorname{Orc}(A)=1$ since $\operatorname{Prim}(A)$ is homeomorphic to an open subset of $\operatorname{Prim}(M(A))$. Furthermore, since $M(A)$ is unital, $H_{x}$ is 2-primal for all $x \in \beta X_{\phi}$ [40, Corollary 2.7] and so $J_{x}$ is locally modular for all $x \in X_{\phi}$ by Theorem 4.6.

(b) $\Rightarrow$ (c). This follows from Proposition 4.5.

(c) $\Rightarrow$ (a). Suppose that (c) holds. Since $\operatorname{Orc}(A)=1, J_{x}$ is 2-primal for all $x \in X_{\phi}$ by Theorem 3.3(c). Hence, as in the proof of Theorem 4.6, $H_{x}=\tilde{J}_{x}$ is 2-primal for all $x \in X_{\phi}=\operatorname{Glimm}(A)$ by Lemma 4.1 and so every Glimm ideal of $\left.M(A)\right)$ is 2-primal by [6, Corollary 3.3].

As noted before Proposition 4.5 , any $C^{*}$-algebra $A$ may be regarded as a $\mathrm{C}(\mathrm{X})$-algebra where $X$ is the Stone-Cech compactification of $\operatorname{Glimm}(A)$ and the base map $\phi$ is the complete regularization map. The corresponding structure map $\mu: C(X) \rightarrow Z(M(A))$ satisfies $\mu(h)=$ $\theta_{A}(h \circ \phi)$ where $\theta_{A}: C^{b}(\operatorname{Prim}(A)) \rightarrow Z(M(A))$ is the Dauns-Hofmann isomorphism (see [10, p. 74]). In this case, the algebra $Z^{\prime}(A):=\mu(C(X)) \cap A$ (see [10, Section 2] and [12, Section $2]$ ) coincides with $Z(A)$. To see this, let $z \in Z(A)$ and let $f=\theta_{A}^{-1}(z) \in C^{b}(\operatorname{Prim}(A))$. By the definition of the complete regularization topology on $\operatorname{Glimm}(A)$, there exists $g \in$ $C^{b}(\operatorname{Glimm}(A))$ such that $f=g \circ \phi$. Let $h \in C(X)$ be the unique extension of $g$. Then

$$
\mu(h)=\theta_{A}(h \circ \phi)=\theta_{A}(g \circ \phi)=\theta_{A}(f)=z .
$$

We now introduce the following notation. Let $A$ be a $\mathrm{C}^{*}$-algebra with centre $Z(A)$ and let $\phi$ be the complete regularization map for $\operatorname{Prim}(A)$. Set $U_{\phi}=\left\{x \in X_{\phi}: J_{x} \nsupseteq Z(A)\right\}$ and let $W_{\phi}=X_{\phi} \backslash U_{\phi}$. Then $U_{\phi}$ is an open subset of $X_{\phi}$ (see [12, Section 2] and [10, Section $2]$ ). Recall that a $\mathrm{C}^{*}$-algebra $A$ is said to be quasi-central if no primitive ideal of $A$ contains $Z(A)$. If $A$ is quasi-central then $U_{\phi}=X_{\phi}(=\operatorname{Glimm}(A))$ and so every Glimm ideal of $A$ is locally modular (see [12, Section 3]).

Corollary 4.8. Let $A$ be a separable $C^{*}$-algebra with $\operatorname{Orc}(A)=1$ and suppose that every Glimm ideal of $A$ is locally modular.

(i) Suppose that $Z(A)=\{0\}$. Then $A$ is a direct sum of primitive $C^{*}$-algebras.

(ii) Suppose that $\operatorname{Prim}(A)$ is a $T_{1}$-space. Then $A=B \oplus C$ where $B$ is quasi-central and $C$ is a direct sum of simple $C^{*}$-algebras.

Proof. (i) Let $\phi$ denote the complete regularization map for $\operatorname{Prim}(A)$. By Proposition 4.5, $H_{x}$ is strictly closed for all $x \in X_{\phi}$. Since $Z(A)=\{0\}, X_{\phi}$ is discrete by [12, Corollary 4.4(ii)]. Let $x \in X_{\phi}$. By Theorem 3.3(c), $J_{x}$ is a 2-primal ideal of $A$. Suppose that $K$ and $L$ are (closed two-sided) ideals of $A$ such that $K L \subseteq J_{x}$. Using the canonical correspondence between open subsets of $\operatorname{Prim}(A)$ and ideals of $A$, we have

$$
\operatorname{Prim}(K) \cap \operatorname{Prim}(L) \subseteq \operatorname{Prim}\left(J_{x}\right)=\operatorname{Prim}(A) \backslash H(x) .
$$

Since $H(x)$ is a clopen subset of $\operatorname{Prim}(A), \operatorname{Prim}(K) \cap H(x)$ and $\operatorname{Prim}(L) \cap H(x)$ are disjoint open subsets of $\operatorname{Prim}(A)$ and so one or other is contained in $\operatorname{Prim}\left(J_{x}\right)$ by the 2-primality of $J_{x}$. Hence either $\operatorname{Prim}(K)$ or $\operatorname{Prim}(L)$ is contained in $\operatorname{Prim}\left(J_{x}\right)$ and so $J_{x}$ is a prime ideal of $A$. Since $A$ is separable, $J_{x}$ is a primitive ideal of $A[37,4.3 .6]$. Hence $A$ is a direct sum of primitive $C^{*}$-algebras.

(ii) Let $x \in X_{\phi}$. Since $\operatorname{Orc}(A)=1$ and $J_{x}$ is locally modular, it follows from [12, Corollary 6.3] that either $x \in U_{\phi}$ or $H(x)$ has non-empty interior. In the latter case, there exists 
a separated point $M$ of $\operatorname{Prim}(A)$ in the interior of $H(x)[19,3.9 .4]$. But $M$ is a maximal ideal since $\operatorname{Prim}(A)$ is a $T_{1}$-space, and thus the singleton $\{M\}$ is a $\sim$-class in $\operatorname{Prim}(A)$ (by definition of a separated point). But $\operatorname{Orc}(A)=1$ and hence $H(x)=\{M\}$ by Theorem 3.3(c) and so $M=J_{x}$. Since $\{M\}$ is a clopen subset of $\operatorname{Prim}(A)$, its characteristic function is continuous and so $x$ is an isolated point in $X_{\phi}$. It follows that $W_{\phi}$ is a discrete open subset of $X_{\phi}$. Set $B=\operatorname{ker} W_{\phi}$ and $C=\operatorname{ker} U_{\phi}$. Then $B$ is quasi-central and $C$ is a direct sum of simple $\mathrm{C}^{*}$-algebras and $A=B \oplus C$.

If $A$ is as in Corollary 4.8 but $\operatorname{Prim}(A)$ is not a $T_{1}$-space then $A$ can have more complicated structure, see [12, Example 6.4(i)].

As Corollary 4.8 shows, the local modularity condition in Corollary 4.7(b) is a restrictive one. However, if $A=C^{*}(G)$ where $G$ is an [SIN] group then $A$ has a central approximate identity (see [36] and [33, Section 1]). Hence $A$ is quasi-central and so every Glimm ideal of $A$ is locally modular.

\section{Applications to norms of elementary operators}

In this section we give some applications of the results of the previous section to norms of elementary operators. We begin with the definitions of the constants $K$ and $K_{s}$ associated with inner derivations, see [25], [3]. Let $A$ be a $\mathrm{C}^{*}$-algebra and let $a \in A$. Then a simple application of the triangle inequality shows that

$$
\|D(a, A)\| \leq 2 d(a, Z(A))
$$

where $D(a, A)$ is the inner derivation generated by $a$ and $d(a, Z(A))$ is the distance from $a$ to $Z(A)$, the centre of $A$. Define $K(A)$ to be the smallest number in $[0, \infty]$ such that

$$
K(A)\|D(a, A)\| \geq d(a, Z(A))
$$

for all $a \in A$. If the elements $a$ are restricted to being self-adjoint then the corresponding constant is denoted $K_{s}(A)$. Clearly $K_{s}(A) \leq K(A)$, and it follows from (1) that $1 / 2 \leq K_{s}(A)$ unless $A$ is commutative.

It was shown in [40, Theorem 4.4] that if $A$ is a unital non-commutative $\mathrm{C}^{*}$-algebra then $K_{s}(A)=1 / 2$ if and only if $\operatorname{Orc}(A)=1$. If $A$ is a non-unital $C^{*}$-algebra then, as discussed in Section 1, the multiplier algebra $M(A)$ provides the natural setting in which to study inner derivations and their norms. By applying the unital result above to the algebra $M(A)$ and combining with Corollary 4.7, we obtain the following.

Theorem 5.1. Let $A$ be a separable, non-commutative $C^{*}$-algebra. Then $K_{s}(M(A))=1 / 2$ if and only if $\operatorname{Orc}(A)=1$ and every Glimm ideal of $A$ is locally modular.

In a similar direction, it was shown in [41, Theorems 3.2 and 3.3] that for a unital noncommutative $\mathrm{C}^{*}$-algebra $A, K(A)=1 / 2$ if and only if every Glimm ideal of $A$ is 3 -primal. Thus from Theorem 4.6 we obtain the following.

Theorem 5.2. Let $A$ be a separable, non-commutative $C^{*}$-algebra. Then $K(M(A))=1 / 2$ if and only if every Glimm ideal of $A$ is locally modular and 3-primal.

In [6, Section 4] there are several examples of second countable, locally compact groups $G$ such that $K\left(M\left(C^{*}(G)\right)\right)=1 / 2$. 
It was also shown in [41, Theorems 3.3 and 3.4] that if $A$ is a unital $\mathrm{C}^{*}$-algebra then $K(A)=1 / \sqrt{3}$ if and only if every Glimm ideal of $A$ is 2-primal (i.e. $\operatorname{Orc}(A)=1$ ) but $A$ has a Glimm ideal which is not 3-primal. Applying this to $M(A)$, we obtain the following from Theorem 4.6.

Theorem 5.3. Let $A$ be a separable $C^{*}$-algebra. Then $K(M(A))=1 / \sqrt{3}$ if and only if every Glimm ideal of $A$ is locally modular and 2-primal but at least one Glimm ideal of $A$ is not 3-primal.

Proof. Suppose first that $K(M(A))=1 / \sqrt{3}$. Then every Glimm ideal of $M(A)$ is locally modular and 2-primal but at least one is not 3-primal. By Theorem 4.6 (first with $n=2$ and then with $n=3$ ), every Glimm ideal of $A$ is locally modular and 2-primal but at least one is not 3-primal.

Conversely, suppose that every Glimm ideal of $A$ is locally modular and 2-primal but at least one Glimm ideal of $A$ is not 3-primal. Then by Theorem 4.6 (with $n=2$ and $n=3$ ), every Glimm ideal of $M(A)$ is 2-primal but $M(A)$ has at least one Glimm ideal which is not 3-primal. Hence $K(M(A))=1 / \sqrt{3}$.

On the other hand, it was shown in [40, Theorem 4.4] that if $A$ is a unital non-commutative $C^{*}$-algebra then $K_{s}(A)=\operatorname{Orc}(A) / 2$. Thus Corollary 4.7 implies the following.

Theorem 5.4. Let $A$ be a separable $C^{*}$-algebra. Then the following are equivalent:

(i) either $\operatorname{Orc}(A)>1$ or $A$ has a Glimm ideal which is not locally modular;

(ii) $K(M(A)) \geq K_{s}(M(A)) \geq 1$.

For the computation of $K\left(M\left(C^{*}(G)\right)\right)$ for a number of group $\mathrm{C}^{*}$-algebras, see [6] and [7]. It was shown in [6, Theorem 4.8] that if $G$ is a second countable [FD] $]^{-}$-group of type I then either $K\left(M\left(C^{*}(G)\right)\right) \leq 1 / 2$ or $K\left(M\left(C^{*}(G)\right)\right)=1$ and hence, in particular, $K\left(M\left(C^{*}(G)\right)\right) \neq 1 / \sqrt{3}$. This raised the question of finding a locally compact group $G$ such that $K\left(M\left(C^{*}(G)\right)\right)=1 / \sqrt{3}[6$, p. 2052]. By using results of Losert [33], we show below that this is not possible for second countable groups which are amenable or CCR or almost connected. We begin with a lemma.

Lemma 5.5. Let $A$ be a $C^{*}$-algebra of the form $A=B \oplus C$ where $B$ is a separable $C^{*}$-algebra with centre $Z(B)=\{0\}$ and $C$ is a quasi-central, quasi-standard $C^{*}$-algebra. Then either $K(M(A)) \leq 1 / 2$ or $K(M(A)) \geq 1$. Equivalently, $K(M(A)) \neq 1 / \sqrt{3}$.

Proof. If $\operatorname{Orc}(M(A)) \geq 2$ then $K(M(A)) \geq 1$ [40, Theorem 4.4], so we suppose from now on that $\operatorname{Orc}(M(A))=1$. Since $M(A)=M(B) \oplus M(C), \operatorname{Orc}(M(B))=1$ and hence $B$ is a direct sum of primitive $C^{*}$-algebras by Corollary 4.8. Then $\operatorname{Glimm}(B)$ is discrete and $B$ is quasi-standard by $[9$, Theorem 3.4]. We now have that $M(B)$ is quasi-standard [10, Corollary 4.9] and so also is $M(C)$ [10, Corollary 4.10]. It follows that $M(A)$ is quasi-standard and hence $K(M(A)) \leq 1 / 2$ [41, Theorem 3.2]. The final part of the statement follows from the trichotomy in $[41$, p. 569].

For the next result, we recall that if a locally compact group $G$ has a compact normal subgroup $N$ then there is a canonical *-isomorphism $\epsilon_{N}$ from $C^{*}(G / N)$ onto a direct summand of $C^{*}(G)$ such that $\epsilon_{N}$ is a right inverse for the canonical *-homomorphism $\tau_{N}: C^{*}(G) \rightarrow C^{*}(G / N)$ and hence $C^{*}(G)=\operatorname{ker} \tau_{N} \oplus \epsilon_{N}\left(C^{*}(G / N)\right)$. More generally, the 
same holds true if $N$ is a closed normal subgroup with property $\mathrm{T}$ (see [33, Section 2] and [42, Section 3]).

Theorem 5.6. Let $A=C^{*}(G)$ where $G$ is a second countable locally compact group which is either (i) amenable or (ii) CCR or (iii) almost connected. Then either $K(M(A)) \leq 1 / 2$ or $K(M(A)) \geq 1$. Equivalently, $K(M(A)) \neq 1 / \sqrt{3}$.

Proof. Since $G$ is second countable, $A$ is separable and hence so is any $C^{*}$-subalgebra. If $Z(A)=\{0\}$ then the result follows from Lemma 5.5 with $C=\{0\}$. So we assume from now on that $Z(A) \neq\{0\}$.

(i) Suppose that $G$ is amenable. By [33, Corollary 1.2], $G$ is an IN-group. As discussed in [33, proof of Corollary 1.3] (see also [32, proof of Proposition 1.2]), $G$ has a compact normal subgroup $K$ such that $G / K$ is a SIN-group and

$$
Z\left(L^{1}(G)\right)=\epsilon_{K}\left(Z\left(L^{1}(G / K)\right)\right) \subseteq \epsilon_{K}\left(C^{*}(G / K)\right) .
$$

Since $G$ is amenable, $Z(A)$ is the norm-closure of $Z\left(L^{1}(G)\right)$ [33, Theorem 1.1] and hence is contained in $\epsilon_{K}\left(C^{*}(G / K)\right)$. Writing $B=\operatorname{ker} \tau_{K}$, we have $Z(B)=\{0\}$. Since $G / K$ is an amenable SIN-group, $C^{*}(G / K)$ is quasi-central [33, Corollary 1.3] and quasi-standard [27] and hence the same holds for $C:=\epsilon_{K}\left(C^{*}(G / K)\right)$. The result follows from Lemma 5.5.

(ii) Suppose instead that $G$ is a CCR group. By [33, Theorem 3.1], $G$ has a closed normal subgroup $N$ with property T such that $G / N$ is an amenable SIN-group and $\epsilon_{N}\left(Z\left(C^{*}(G / N)\right)\right)=$ $Z\left(C^{*}(G)\right)$. With $B:=\operatorname{ker} \tau_{N}$ and $C:=\epsilon_{K}\left(C^{*}(G / N)\right)$ we have $Z(B)=\{0\}$ and, as before, $C$ is quasi-central and quasi-standard. The result follows from Lemma 5.5.

(iii) Finally, suppose instead that $G$ is almost connected. By [33, Theorem 5.1 and its proof], $G$ has a closed normal subgroup $N$ with property $\mathrm{T}$ such that $G / N$ is an amenable IN-group and $\epsilon_{N}\left(Z\left(C^{*}(G / N)\right)\right)=Z\left(C^{*}(G)\right) \neq\{0\}$. Arguing as in (i) (with $G$ replaced by $G / N)$, we obtain $C^{*}(G / N)=B \oplus C$ where $Z(B)=\{0\}$ and $C$ is quasi-central and quasi-standard. The result follows by applying Lemma 5.5 to the decomposition $C^{*}(G)=$ $\left(\operatorname{ker} \tau_{N} \oplus \epsilon_{N}(B)\right) \oplus \epsilon_{N}(C)$.

Now let $A$ be a $C^{*}$-algebra and let $Z$ be the centre of $M(A)$. Let $A \otimes_{h} A$ denote the Haagerup tensor product. Then the central Haagerup tensor product $A \otimes_{Z, h} A$ is the quotient of $A \otimes_{h} A$ by the subspace $J_{A}$ defined to be the closure of the span of elements of the form $a z \otimes b-a \otimes z b(a, b \in A, z \in Z)$. Let $\mathrm{CB}(A)$ be the space of completely bounded maps on $A$ and let $\theta$ be the contractive linear map from $A \otimes_{h} A \rightarrow \mathrm{CB}(A)$ defined on elementary tensors by

$$
\theta\left(\sum_{i=1}^{n} a_{i} \otimes b_{i}\right)(x)=\sum_{i=1}^{n} a_{i} x b_{i} \quad(x \in A) .
$$

Thus $\theta(u)$ is an elementary operator whenever $u$ is an elementary tensor. Furthermore $J_{A} \subseteq \operatorname{ker} \theta$, so $\theta$ induces a contractive map $\theta_{Z}: A \otimes_{Z, h} A \rightarrow \operatorname{CB}(A)$ where $\theta_{Z}\left(u+J_{A}\right)=\theta(u)$ $\left(u \in A \otimes_{h} A\right)$. It was shown in [14, Theorem 3.8] that $\theta_{Z}$ is injective if and only if every Glimm ideal of $A$ is 2-primal and that $\theta_{Z}$ is isometric if and only if every Glimm ideal of $A$ is primal.

If $A$ is non-unital then we can also consider the extended map $\bar{\theta}_{Z}: M(A) \otimes_{Z, h} M(A) \rightarrow$ $\mathrm{CB}(M(A))$ and its restriction $\Theta_{Z}: M(A) \otimes_{Z, h} M(A) \rightarrow \mathrm{CB}(A)$ defined by

$$
\Theta_{Z}\left(u+J_{M(A)}\right)=\left.\bar{\theta}_{Z}\left(u+J_{M(A)}\right)\right|_{A} \quad\left(u \in M(A) \otimes_{h} M(A)\right) .
$$


It was shown in [13, Corollary 9] that $\Theta_{Z}$ is isometric if and only if every Glimm ideal of $M(A)$ is primal. Thus Theorem 4.6 implies the following.

Theorem 5.7. Let $A$ be a separable $C^{*}$-algebra. The canonical contraction

$$
\Theta_{Z}: M(A) \otimes_{Z, h} M(A) \rightarrow \mathrm{CB}(A)
$$

is isometric if and only if every Glimm ideal of $A$ is locally modular and primal.

Theorem 4.2 requires a separability hypothesis, or something similar, and so the theorems thus far in this section are valid only for separable $\mathrm{C}^{*}$-algebras. The problem of determining the $\sigma$-unital $\mathrm{C}^{*}$-algebras $A$ for which $\operatorname{Orc}(M(A))=1$ seems to be formidable, even if one makes the further assumption that $A$ is quasi-standard. If $A$ has enough non-unital quotients, however, then this problem can be solved [6, Theorem 3.6]. Theorem 5.9 will supplement that result. We begin with a proposition which augments the results in [31] and [18] concerning the relationship between modular ideals and non-trivial centre. It shows that the density hypothesis in [6, Theorem 3.6] can be replaced by the assumption of trivial centre.

Proposition 5.8. Let $A$ be a $\sigma$-unital quasi-standard $C^{*}$-algebra. The following conditions are equivalent:

(i) the centre $Z(A)=\{0\}$;

(ii) the set $S=\{P \in \operatorname{Prim}(A): A / P$ is non-unital $\}$ is dense in $\operatorname{Prim}(A)$.

Proof. (ii) $\Rightarrow$ (i) This is an elementary consequence of the lower semi-continuity of norm functions (see, for example, [31, Section 1] and [6, Proposition 2.1]).

(i) $\Rightarrow$ (ii). Note that if $P \in \operatorname{Prim}(A)$ and $A / P$ is unital then the assignment $a+P \rightarrow a+\tilde{P}$ $(a \in A)$ defines a ${ }^{*}$-isomorphism of $A / P$ onto $M(A) / \tilde{P}$ because the image is a unital essential ideal of $M(A) / \tilde{P}$. Let $u$ be a strictly positive element in $A$ with $\|u\|=1$. Suppose that the set $S$ is not dense in $\operatorname{Prim}(A)$ and that $W$ is a non-empty open subset of $\operatorname{Prim}(A)$ with $A / P$ unital for all $P \in W$. The norm function $P \mapsto\|(1-u)+\tilde{P}\|(P \in \operatorname{Prim}(A))$ is lower semi-continuous on the Baire space $\operatorname{Prim}(A)$ and thus there is a dense subset of $\operatorname{Prim}(A)$ consisting of points of continuity of this norm function (see the proof of [19, Appendice B18]). Let $Q \in W$ be such a point of continuity. Since $A / Q$ is unital, $\|(1-u)+\tilde{Q}\|<1$ and hence there is an open neighbourhood $W^{\prime}$ of $Q$ in $\operatorname{Prim}(A)$ such that $\|(1-u)+\tilde{P}\|<(1+\alpha) / 2$ for all $P \in W^{\prime}$, where $\alpha=\|(1-u)+\tilde{Q}\|$.

Now let $R \in W^{\prime}$ and let $S \in \operatorname{Prim}(A)$ with $R \sim S$. Then $\|(1-u)+\tilde{S}\| \leq(1+\alpha) / 2$ for otherwise the set $\{P \in \operatorname{Prim}(A):\|(1-u)+\tilde{P}\|>(1+\alpha) / 2\}$ is an open neighbourhood of $S$ disjoint from $W^{\prime}$, contradicting the fact that $S \sim R$. Let $W^{\prime \prime}$ be the $\sim$-saturation of $W^{\prime}$ and let $Y=\phi\left(W^{\prime \prime}\right)$, where $\phi$ is the complete regularization map for $\operatorname{Prim}(A)$. Then $Y$ is an open subset of $X_{\phi}=\operatorname{Glimm}(A)$ since $A$ is quasi-standard (see [9, Proposition 3.2 and Theorem 3.3]).

Let $f:[0,1] \rightarrow[0,1]$ be a continuous function such that $f(0)=0$ and $f([(1-\alpha) / 2,1])=$ $\{1\}$. Let $v=f(u) \in A$. For all $P \in W^{\prime \prime}$, the spectrum of $u+\tilde{P}$ is contained in $[(1-\alpha) / 2,1]$ and so $\|(1-v)+\tilde{P}\|=0$ which implies that $v+P$ is the identity of $A / P$. Let $h: X_{\phi} \rightarrow[0,1]$ be a non-zero continuous function vanishing off $Y$ and let $z=\theta_{A}(h \circ \phi) \in Z(M(A))$, where $\theta_{A}: C^{b}(\operatorname{Prim}(A)) \rightarrow Z(M(A))$ is the Dauns-Hofmann isomorphism. If $P \in W^{\prime \prime}$ then $z v+P=h(\phi(P))(v+P)$ and if $P \in \operatorname{Prim}(A) \backslash W^{\prime \prime}$ then $z v+P=h(\phi(P))(v+P)=0$ because $\phi(P) \notin Y$. Hence $z v$ is a non-zero central element in $A$. 
Recall that a completely regular Hausdorff space $X$ is an F-space if disjoint cozero sets in $X$ can be separated by disjoint zero sets.

Theorem 5.9. Let $A$ be a $\sigma$-unital quasi-standard $C^{*}$-algebra and suppose that the centre $Z(A)=\{0\}$. Let $\Theta_{Z}: M(A) \otimes_{Z, h} M(A) \rightarrow \mathrm{CB}(A)$ be the canonical contraction. Then the following are equivalent:

(i) $\operatorname{Glimm}(A)$ is an F-space;

(ii) $\operatorname{Orc}(M(A))=1$;

(iii) $K(M(A))=K_{s}(M(A))=1 / 2$;

(iv) every Glimm ideal of $M(A)$ is primal;

(v) the map $\Theta_{Z}$ is isometric;

(vi) the map $\Theta_{Z}$ is injective.

Proof. The equivalence of (i) - (iv) follows from [6, Theorem 3.6] and Proposition 5.8. The implication (iv) $\Rightarrow(\mathrm{v})$ follows from [13, Corollary 9] while (v) $\Rightarrow($ vi) is trivial. Now suppose that (vi) holds. Let $u \in M(A) \otimes_{h} M(A)$ and suppose that $\bar{\theta}_{Z}\left(u+J_{M(A)}\right)=0$. Then $\Theta_{Z}\left(u+J_{M(A)}\right)=\left.\bar{\theta}_{Z}\left(u+J_{M(A)}\right)\right|_{A}=0$, so $u \in J_{M(A)}$ by assumption. Hence $\bar{\theta}_{Z}$ is injective, so every Glimm ideal of $M(A)$ is 2-primal by [14, Theorem 3.8]. Thus (ii) holds.

Let $A$ be a $\sigma$-unital quasi-standard $\mathrm{C}^{*}$-algebra with $Z(A)=\{0\}$. Then Theorem 5.9 shows that every Glimm ideal of $M(A)$ is primal if and only if $\operatorname{Glimm}(A)$ is an F-space. On the other hand, $M(A)$ satisfies the stronger condition of being quasi-standard if and only if $\operatorname{Glimm}(A)$ is a basically disconnected space [10, Corollary 4.9].

Notice, in conclusion, that the density of the subset $S$ in Lemma 5.8 implies that if $A$ is a $\sigma$-unital quasi-standard $C^{*}$-algebra with $Z(A)=\{0\}$ then any locally modular Glimm ideal of $A$ must be an isolated point in $\operatorname{Glimm}(A)$. On the other hand, there exist F-spaces such as $\beta \mathbb{N} \backslash \mathbb{N}$ which have no isolated points. Thus the local modularity condition of Corollary 4.7 loses its significance for the problem of characterizing $\operatorname{Orc}(M(A))=1$ when the separability assumption on $A$ is dropped.

\section{Appendix: Proof of lemma 3.1}

Lemma 3.1 [15, Vol.2: Exercise 15(b) on p.244] Let $X$ be a locally compact, Hausdorff, $\sigma$-compact space and let $R$ be an equivalence relation on $X$ whose graph is closed in $X \times X$. Then $X / R$ is Hausdorff and normal.

Proof. Let $x \in X$. Since the graph of $R$ is closed, the equivalence class $[x]=\{y \in X: y R x\}$ is closed in $X$. It follows that singleton subsets of $X / R$ are closed, i.e. $X / R$ is $T_{1}$. So it suffices to prove that $X / R$ is normal. Let $q: X \rightarrow X / R$ be the quotient map.

First note that if $Y$ is a compact subset of $X$ then $q(Y)$ is closed. To see this, let $Z=q^{-1}(q(Y))$ and let $\left(x_{\alpha}\right)$ be a net in $Z$ converging to a limit $x \in X$. Then for each $\alpha$ there exists $y_{\alpha} \in Y$ such that $x_{\alpha} R y_{\alpha}$. Using the compactness of $Y$, we may obtain a subnet $\left(y_{\beta}\right)$ of $\left(y_{\alpha}\right)$ such that $\left(y_{\beta}\right)$ converges to a limit $y \in Y$. But then $x R y$ since the graph of $R$ is closed, so $x \in Z$. Hence $q(Y)$ is closed by definition of the quotient topology.

Since $X$ is $\sigma$-compact and locally compact, standard arguments lead to the existence of a sequence $\left(U_{n}\right)$ of open subsets of $X$ such that $\bar{U}_{n}$ is a compact subset of $U_{n+1}$ for all $n \geq 1$ and $X=\bigcup_{n=1}^{\infty} U_{n}$. For $n \geq 1$, let $X_{n}=\bar{U}_{n}$ and let $R_{n}$ be the restriction of $R$ to $X_{n}$, that is $R_{n}=R \cap\left(X_{n} \times X_{n}\right)$. Since the graph of $R$ is closed, the graph of $R_{n}$ is closed in $X_{n} \times X_{n}$. 
By [15, Vol.1, Prop.8 on p.105], $X_{n} / R_{n}$ is compact and Hausdorff and hence normal. Let $q_{n}: X_{n} \rightarrow X_{n} / R_{n}$ be the quotient map and let $i_{n}: X_{n} / R_{n} \rightarrow X / R$ be given by $i_{n}([x])=q(x)$ $\left(x \in X_{n}\right)$. Then $i_{n}$ is a bijection between $X_{n} / R_{n}$ and $q\left(X_{n}\right)$.

We now show that $i_{n}$ is a homeomorphism between $X_{n} / R_{n}$ and $q\left(X_{n}\right)$. Note that $q\left(X_{n}\right)$ is closed by the work of the second paragraph above. Let $W$ be a subset of $q\left(X_{n}\right)$. We claim that the following is true: $W$ is closed if and only if $Z:=q^{-1}(W)$ is closed if and only if $Z \cap X_{n}$ is closed if and only if $q_{n}\left(Z \cap X_{n}\right)$ is closed. The first and third equivalences follow from the definition of the quotient topology together with the fact that $Z \cap X_{n}$ is an $R_{n}$-saturated subset of $X_{n}$. For the second equivalence, one direction is trivial. For the other direction, note that if $Z \cap X_{n}$ is closed then it is compact and hence $Z=q^{-1}\left(q\left(Z \cap X_{n}\right)\right)$ is closed by the second paragraph above. Since $i_{n} \circ q_{n}\left(Z \cap X_{n}\right)=W$ it follows that $i_{n}$ is a homeomorphism. Hence $q\left(X_{n}\right)$ is normal for all $n \geq 1$.

Next note that a subset $W$ of $X / R$ is closed if (and only if) $W \cap q\left(X_{n}\right)$ is a closed subset of $q\left(X_{n}\right)$ for all $n \geq 1$. Indeed, suppose that $W \cap q\left(X_{n}\right)$ is closed for all $n \geq 1$ and let $\left(x_{\alpha}\right)$ be a net in $Z:=q^{-1}(W)$ converging to a limit $x \in X$. Then $x \in U_{m}$ for some $m$ and hence eventually $x_{\alpha} \in U_{m} \subseteq X_{m}$. Thus eventually $q\left(x_{\alpha}\right) \in W \cap q\left(X_{m}\right)$ and $q\left(x_{\alpha}\right) \rightarrow q(x)$. Since $W \cap q\left(X_{m}\right)$ is a closed subset of $q\left(X_{m}\right)$, it follows that $q(x) \in W \cap q\left(X_{m}\right)$ and hence that $x \in Z$. Thus $Z$ is closed, so $W$ is closed. By taking complements, it follows that a subset $W$ of $X / R$ is open if and only if $W \cap q\left(X_{n}\right)$ is an open subset of $q\left(X_{n}\right)$ for all $n \geq 1$.

Now let $A$ and $B$ be disjoint closed subsets of $X / R$. For each $n \geq 1$, set $A_{n}=A \cap q\left(X_{n}\right)$ and $B_{n}=B \cap q\left(X_{n}\right)$. Then $A_{1}$ and $B_{1}$ are closed and disjoint so, working in $q\left(X_{1}\right)$, by two applications of normality there exist disjoint closed neighbourhoods $C_{1}$ and $D_{1}$ of $A_{1}$ and $B_{1}$ respectively. Then $A_{2} \cup C_{1}$ and $B_{2} \cup D_{1}$ are disjoint closed subsets of $q\left(X_{2}\right)$ so, working in $q\left(X_{2}\right)$, there exist disjoint closed neighbourhoods $C_{2}$ and $D_{2}$ of $A_{2} \cup C_{1}$ and $B_{2} \cup D_{1}$ respectively. Proceeding inductively, for each $n \geq 2$ we may find in $q\left(X_{n}\right)$ disjoint closed neighbourhoods $C_{n}$ and $D_{n}$ of $A_{n} \cup C_{n-1}$ and $B_{n} \cup D_{n-1}$ respectively. For each $n \geq 1$, let $V_{n}$ be the interior of $C_{n}$ in $q\left(X_{n}\right)$ and $W_{n}$ the interior of $D_{n}$ in $q\left(X_{n}\right)$. Set $V=\bigcup_{n=1}^{\infty} V_{n}$ and $W=\bigcup_{n=1}^{\infty} W_{n}$. Since $C_{n}$ and $D_{n}$ both increase with $n$, so too do $V_{n}$ and $W_{n}$. For $N \geq 1$, $V \cap q\left(X_{N}\right)=\bigcup_{n \geq N}\left(V_{n} \cap q\left(X_{N}\right)\right)$ which is open in $q\left(X_{N}\right)$. Hence $V$, and similarly $W$, is open in $X$ by the previous paragraph. Furthermore for $m, n \geq 1, V_{m} \cap W_{n} \subseteq C_{m} \cap D_{n}=\emptyset$, and hence $V$ and $W$ are disjoint. Since $A_{n} \subseteq V_{n}$ and $B_{n} \subseteq W_{n}$ for each $n \geq 1$, we see that $A \subseteq V$ and $B \subseteq W$. Hence $X / R$ is normal as required.

\section{REFERENCES}

[1] C. A. AKEMANN, G. K. PEDERSEN and J. TOMIYAMA, 'Multipliers of C*-algebras', J. Funct. Anal., 13 (1973) 277-301.

[2] P. ARA and M. MATHIEU, Local Multipliers of $C^{*}$-Algebras, Springer, London, 2003.

[3] R. J. ARCHBOLD, 'On the norm of an inner derivation of a $\mathrm{C}^{*}$-algebra', Math. Proc. Camb. Phil. Soc., 84 (1978) 273-291.

[4] R. J. ARCHBOLD and C. J. K. BATTY, 'On factorial states of operator algebras, III', J. Operator Theory, 15 (1986) 53-81.

[5] R. J. ARCHBOLD and E. KANIUTH, 'Simply connected nilpotent Lie groups with quasi-standard C*-algebras', Proc. Amer. Math. Soc., 125 (1997) 2733-2742.

[6] R. J. ARCHBOLD, E. KANIUTH, and D. W. B. SOMERSET, 'Norms of inner derivations for multiplier algebras of C*-algebras and group C*-algebras', J. Funct. Anal., 262 (2012) 2050-2073.

[7] R. J. ARCHBOLD, E. KANIUTH, and D. W. B. SOMERSET, 'Norms of inner derivations for multiplier algebras of $\mathrm{C}^{*}$-algebras and group $\mathrm{C}^{*}$-algebras, II', submitted. 
[8] R. J. ARCHBOLD, E. KANIUTH, G. SCHLICHTING, and D. W. B. SOMERSET, 'On the topology of the dual of a nilpotent Lie group', Math. Proc. Camb. Phil. Soc., 125 (1999) 269-293.

[9] R. J. ARCHBOLD and D. W. B. SOMERSET, 'Quasi-standard C*-algebras', Math. Proc. Camb. Phil. Soc., 107 (1990) 349-360.

[10] R. J. ARCHBOLD and D. W. B. SOMERSET, 'Multiplier algebras of $C_{0}(X)$-algebras', Münster J. Math., 4 (2011) 73-100.

[11] R. J. ARCHBOLD and D. W. B. SOMERSET, 'Ideals in the multiplier and corona algebras of a $C_{0}(X)$-algebra', J. London Math. Soc., (2) 85 (2012) 365-381.

[12] R. J. ARCHBOLD and D. W. B. SOMERSET, 'Spectral synthesis in the multiplier algebra of a $C_{0}(X)$-algebra', Quart. J. Math. (2013); doi: 10.1093/qmath/has048.

[13] R. J. ARCHBOLD, D. W. B. SOMERSET, and R. M. TIMONEY, 'On the central Haagerup tensor product and completely bounded mappings of a C*-algebra', J. Funct. Anal., 226 (2005) 406-428.

[14] R. J. ARCHBOLD, D. W. B. SOMERSET, and R. M. TIMONEY, 'Completely bounded mappings and simplicial complex structure in the primitive ideal space of a $\mathrm{C}^{*}$-algebra', Trans. Amer. Math. Soc., 361 (2009) 1397-1427.

[15] N. BOURBAKI, General Topology, 2 vols, Addison-Wesley, Paris, 1966.

[16] L. G. BROWN, 'On higher real and stable ranks for CCR C*-algebras', arXiv:0708.3072.

[17] J. DAUNS and K. H. HOFMANN, Representations of Rings by Continuous Sections, Memoir 83, American Math. Soc., Providence, R.I., 1968.

[18] C. DELAROCHE, Sur les centres de C*-algebres, II, Bull. Sci. Math. 92 (1968) 111-128.

[19] J. DIXMIER, C*-algebras, North-Holland, Amsterdam, 1977.

[20] J. M. G. FELL, 'The dual spaces of C*-algebras', Trans. Amer. Math. Soc., 94 (1960) 365-403.

[21] J. M. G. FELL, 'The structure of algebras of operator fields', Acta Mathematica, 106 (1961) 233-280.

[22] J. M. G. FELL, 'A Hausdorff topology for the closed sets of a locally compact non-Hausdorff space', Proc. Amer. Math. Soc., 13 (1962) 472-476.

[23] L. GILLMAN and M. JERISON, Rings of Continuous Functions, Van Nostrand, New Jersey, 1960.

[24] J. GLIMM, 'A Stone-Weierstrass theorem for C*-algebras', Ann. of Math., 72 (1960) 216-244.

[25] R. V. KADISON, E. C. LANCE and J. R. RINGROSE, 'Derivations and automorphisms of operator algebras, II', J. Funct. Anal., 1 (1967) 204-221.

[26] E. KANIUTH, 'Minimal primal ideal spaces and norms of inner derivations of tensor products of C*-algebras', Math. Proc. Camb. Phil. Soc., 119 (1996) 297-308.

[27] E. KANIUTH, G. SCHLICHTING, and K. TAYLOR, 'Minimal primal and Glimm ideal spaces of group C*-algebras', J. Funct. Anal., 130 (1995) 45-76.

[28] I. KAPLANSKY, 'The structure of certain operator algebras', Trans. Amer. Math. Soc., 70 (1951) 219-255.

[29] K. KURATOWSKI, Topology, vol.1, Academic Press, New York, 1966.

[30] A. LAZAR, 'Quotient spaces determined by algebras of continuous functions', Israel J. Math., 179 (2010) $145-155$.

[31] A. LAZAR, 'Centers of $C^{*}$-algebras rich in modular ideals', preprint.

[32] J. LIUKKONEN and R. MOSAK, 'Harmonic analysis and centers of group algebras', Trans. Amer. Math. Soc., 195 (1974) 147-163.

[33] V. LOSERT, 'On the center of group C*-algebras' J. Reine Angew. Math., 554 (2003) 105-138.

[34] B. MAGAJNA, 'Uniform approximation by elementary operators', Proc. Edin. Math. Soc., 52 (2009) 731-749.

[35] O. A. NIELSEN, 'Unitary representations and coadjoint orbits of low-dimensional Lie groups', Queen's Papers in Pure Appl. Math., 63 (1983).

[36] R. D. MOSAK, 'Central functions in group algebras', Proc. Amer. Math. Soc., 29 (1971) 613-616.

[37] G. K. PEDERSEN, C*-algebras and their Automorphism Groups, Academic Press, London, 1979.

[38] N. C. PHILLIPS, 'Recursive subhomogeneous algebras', Trans. Amer. Math. Soc., 359 (2007) 45954623.

[39] R. R. SMITH and D. P. WILLIAMS, 'Separable injectivity for C*-algebras', Indiana Math. J., 37 (1988) 111-133. 
[40] D. W. B. SOMERSET, 'The inner derivations and the primitive ideal space of a $\mathrm{C}^{*}$-algebra', J. Operator Theory, 29 (1993) 307-321.

[41] D. W. B. SOMERSET, 'Inner derivations and primal ideals of $\mathrm{C}^{*}$-algebras', J. London Math. Soc., (2) 50 (1994) $568-580$.

[42] A. VALETTE, 'Minimal projections, integrable representations and property (T), Arch. Math. (Basel), 43 (1984) 397-406.

[43] R. C. WALKER, The Stone-Cech Compactification, Springer-Verlag, Berlin, 1974.

Institute of Mathematics, University of Aberdeen, King's College, Aberdeen AB24 3Ue, Scotland, United Kingdom

E-mail address: r.archbold@abdn.ac.uk

Institute of Mathematics, University of Aberdeen, King's College, Aberdeen AB24 3UE, Scotland, United Kingdom

E-mail address: somerset@quidinish.fsnet.co.uk 